\title{
Interdependent Preference Models as a Theory of Intentions
}

\author{
Faruk Gul \\ Department of Economics \\ Princeton University \\ Princeton, New Jersey 08540 \\ email: fgul@princeton.edu \\ Wolfgang Pesendorfer \\ Department of Economics \\ Princeton University \\ Princeton, New Jersey 08540 \\ corresponding author; email: pesendor@princeton.edu
}

March 2016

C 2016. This manuscript version is made available under the Elsevier user license

http://www.elsevier.com/open-access/userlicense/1.0/ 


\begin{abstract}
We provide a preference framework for situations in which "intentions matter." A behavioral type describes the individual's observable characteristics and the individual's personality. We define a canonical behavioral type space and provide a condition that identifies collections of behavioral types that are equivalent to components of the canonical type space. We also develop a reciprocity model within our framework and show how it enables us to distinguish between strategic (or instrumental) generosity and true generosity.
\end{abstract}

JEL codes: D110

Keywords: interdependent preferences, reciprocity. 


\section{Introduction}

In many economic settings, knowing the physical consequences of the interaction is not enough to determine its utility consequences. For example, Blount (1995) observes that experimental subjects may reject an unfair division when another subject willingly proposes it and yet might accept it when the other subject is forced to propose it. Hence, individuals care not just about physical consequences but also about the intentions of those around them. In this paper, we develop a framework for modeling intentions and how they affect others' behavior.

We call our descriptions of intentions interdependent preference models (IPMs). In an IPM, a person's ranking of social outcomes depends on the characteristics and personalities of others. Characteristics are attributes such as the individual's wealth, education or gender. Personalities describe how preferences respond to the characteristics and personalities of others. Thus, personality defines a person's altruism, his desire to conform, his willingness to reciprocate or his inclination to be spiteful. To understand how our theory works, consider the following example:

Two individuals can either receive a gift or a pay a penalty. There are four possible outcomes: each person either receives a gift or pays a penalty. Both the spiteful preference, $S$, and the generous preference, $G$, rank receiving a gift above paying a penalty, but $S$ prefers that the opponent pays a penalty whereas $G$ prefers that the opponent receives a gift. $S$ and $G$ are the only possible preferences.

There are 3 possible types for each person. Each type has the same characteristic and therefore types differ only in their personalities. Type 1 has the spiteful preference irrespective of the opponent's type and type 3 has the generous preference irrespective of the opponent's type. Type 2 has the generous preference unless the opponent is type 1. The table below summarizes the mapping from type profiles to preference profiles:

$\begin{array}{cccc} & 1 & 2 & 3 \\ 1 & S, S & S, S & S, G \\ 2 & S, S & G, G & G, G \\ 3 & G, S & G, G & G, G\end{array}$

Example 1: Generous or Spiteful 
We call such a table an IPM. Levine (1998) introduces the first example of an IPM and uses it to address experimental evidence in centipede, ultimatum and public goods experiments.

Three features of IPMs are noteworthy: first, a type describes relevant personality attributes rather than information. These attributes determine both the person's and his opponent's preferences over outcomes, not their beliefs over an uncertain state of nature. To put it another way, IPMs do not incorporate asymmetric information (or interactive knowledge); they only model interactive preferences. Each entry in the table describes the preference the two individuals would have if they knew the other's type; the IPM does not address the question whether an individual knows the others' type.

Second, an IPM does not describe the available strategic choices; it is not a game. We can study how Persons I and II above would play many different games. We can also use this IPM as the preference model for a competitive economy. Hence, IPMs describe only the preference environment not the institutional setting.

Third, in an IPM, individuals have preferences over physical outcomes and these preferences depend on the persistent personalities and characteristics of everyone involved, not on observed or predicted behavior or beliefs. Hence, the interaction of these fixed personalities determines whether each person is generous or spiteful. Whether or not a person acts generously on a given day or believes the other will act generously is relevant only to the extent that these actions affect the physical outcome.

\subsection{Comparison to Psychological Games}

When an IPM is applied to a specific strategic interaction we must specify the game and the information structure. For example, consider the game in which players must simultaneously decide whether the opponent receives a gift or pays a penalty. Preference types are as described in Example 1. Both players know their own preference type but are uncertain about the opponent's type.

Consider the behavior of type 2: he chooses the gift if he believes the opponent is type 2 or 3 and the penalty if he believes the opponent is type 1. Moreover, the expectation that the opponent chooses the gift indicates good intentions (i.e., the opponent is not type 
1) and is met with a generous response. Thus, the IPMs interpret the intentions of a player as the player's objectives.

Geanakoplos, Pearce and Stacchetti's (GPS) (1989) and the many reciprocity models based on their approach (see, for example, Rabin (1993), Dufwenberg and Kirchsteiger (2004), Falk and Fischbacher (2006), Segal and Sobel (2007) and Battigalli and Dufwenberg (2009)) model preference interactions through a circular definition that permits preferences to depend on the very behavior (or beliefs about behavior) that they induce.

A GPS based analysis of the simultaneous action game posits a utility function that prefers the gift if the opponent is expected to choose the gift but prefers the penalty if the opponent is expected to choose the penalty. The (expectation of) the opponent's action is interpreted as revealing a player's intention in this particular game. Thus, GPS-based models interpret the intentions of a player as the player's behavior in the game.

The GPS-based model has complete information but multiple equilibria. Choosing the appropriate equilibrium, we can rationalize all four possible outcomes. ${ }^{1}$ By contrast, the IPM-based model has incomplete information and, for a generic choice of beliefs, a unique equilibrium. Choosing the appropriate beliefs, all four outcomes can be rationalized as an equilibrium of the IPM-based model as well.

The difference between the two approaches becomes relevant when we vary the circumstances in which the interaction takes place. If players play the game once, the generous action indicates generous intentions in the IPM-based model since it excludes the possibility that the player is type 1 . If the game is repeated, then it may be that no inference can be drawn from a generous action. In that case, type 1 may have an incentive to conceal his type and, as a result, may have a material incentive to act generously. ${ }^{2}$

1 The model has two pure strategy equilibria and a mixed equilibrium. The asymmetric outcomes can be rationalized as the outcome of the mixed equilibrium.

2 The question of what kind of behavior is classified as intentional is the subject of a literature in experimental philosophy. In a well-known paper, Knobe (2003) shows that if an agent takes an action that increases his profit but also has a socially beneficial side-effect, the side-effect is typically classified as non-intentional by subjects. Conversely, if the side-effect is harmful (that is, the profit motive and social consequences are in conflict), the action is classified as intentional. The IPM-based interpretation of intentionality creates a related asymmetry: if it is profitable to take the socially beneficial action, no inference can be made on the type of player - hence nothing is learned about his intentions. Conversely, if the profit motive is in conflict with a socially beneficial action we learn that the decision maker cares more about profit than about social outcomes. Hence, something is learned about his intentions. 
To capture the difference between the one-shot and repeated interactions, GPS-based models must tailor the utility function to the specific circumstances. By contrast, IPMbased models provide a separation between the underlying preference framework (i.e., the IPM) and the particular institution (game, market). Therefore, the same IPM can be applied in any economic model.

Because IPMs afford a separation between preferences and institutions, they are well suited for analyzing economic design problems or for comparing institutions. Consider, for example, the (complete information) implementation problem: let $f$ be a social choice rule (or performance criterion) that associates a set of outcomes with each profile, $\theta$, of individual attributes. When is it possible to find a game form $g$ such that all equilibrium outcomes of the game $(g, \theta)$ are among the desirable outcomes $f(\theta)$ for every $\theta$ ? Note that the separation of the preference framework (i.e., the attributes $\theta$ ) from the game form is essential for analyzing such a problem. Without this separation, just stating the implementation problem becomes a formidable task.

More generally, separating preferences from institutions is essential anytime we wish to evaluate a particular institution or assess a specific environmental factor: does the English auction Pareto dominate the Dutch auction? Do prohibitions on resale enhance efficiency? Does greater monitoring increase effort? Questions such as these demand a performance criterion over consequences that can be expressed without reference to specific institutions.

Some departures from standard theory can be accommodated by GPS-based models but fall outside the reach of IPMs. For example, Geanakoplos, Pearce and Stacchetti show how psychological games can model a preference for being surprised or a preference for pandering to others' expectations. Such preferences are best described within the context of the particular interaction and hence are difficult to model as IPMs.

\subsection{What is a personality type?}

In an IPM, a type maps the other person's types to preference profiles. Hence, the definition of a type is circular. Our first objective is to identify a criterion to determine when this circularity is problematic and when it is not; that is, to identify when interdependent preference types can be reduced to preference statements. To see how this can be done, note that in the above example, the preference profile associated with types 1 and 
3 does not depend on the opponent's personality. Type 1 can be identified as the person-

ality that is always spiteful and type 3 can be identified as the personality that is always generous. But once type 1 is identified, type 2 can describe himself as a personality that is spiteful only to the personality that is always spiteful. Hence, in the above example, we can eliminate the circularity by restating each type as a hierarchy of preference statements.

We define the canonical type space for interdependent preferences as sequences of such hierarchical preferences statements. In Theorem 1, we show that each component of the canonical type space is an IPM. However, not every IPM is part of the canonical type space. Theorem 2 provides a simple condition on IPMs, (validity) that guarantees that an IPM is a component of the canonical type space.

\subsection{Two Implications of Validity}

We provide two applications that demonstrate a connection between our validity condition and the identifiability of personality types. The first application considers a population of players who are randomly matched into pairs and then take economic actions that reveal their preferences. Types are drawn from an IPM and commonly known among players. An analyst observes the actions of a sample of players but does not know their types.

We say that types are observable if the analyst can infer the type of the player from a sufficiently rich (but finite) data set. Theorem 3 shows that the types of an IPM are observable if and only if the IPM is valid. Thus, an implication of validity is that types can be identified from observed behavior. Conversely, this identification is not possible if the model is not valid.

In the second application, we consider the problem of a player who wishes to communicate her preference type. We interpret the IPM and its state space as the modeler's convenient representation of the player's possible personalities. Hence, the type space is not a primitive of the model and the statement, "I am type 1" is not meaningful. Instead, if a player wishes to communicate her type, she must do so in terms of the primitives of the model. To formalize this requirement, we develop a notion of communicability in the language of preferences. We show that players can communicate their type in the language of preferences if and only if the model is valid (Theorem 4). 


\subsection{Reciprocity}

As an application of the model, we define reciprocity and identify a class of valid interdependent preference models with reciprocating types. To do this, we consider settings in which preferences can be ranked according to their kindness. That is, we identify each

preference with a real number and interpret higher numbers as kinder preferences. For example, assume $x_{i}$ is $i$ 's consumption and

$$
V^{r}\left(x_{1}, x_{2}\right)=u\left(x_{1}\right)+r u\left(x_{2}\right)
$$

is the utility that person 1 enjoys if $r$ is a preference parameter. The IPM specifies how types determine this parameter. Let $\delta\left(t, t^{\prime}\right) \in[\underline{r}, \bar{r}]$ be the preference of type $t$ when the opponent is type $t^{\prime}$. A higher $r$ is a kinder preference. Type $t$ is nicer than type $t^{\prime}$ if $t$ is kinder than $t^{\prime}$ to every opponent type; a type reciprocates if it is kinder to a nicer opponent. Hence, if $\delta$ is increasing in the first argument, higher types are kinder. Then, if $\delta$ is also increasing in the second argument, all types reciprocate. In Theorems 5 and 6 we characterize two simple classes of reciprocity models.

\subsection{Related Literature}

Our canonical type space provides a foundation for IPMs that is analogous to the Mertens and Zamir (1985)/Brandenburger and Dekel (1993) foundations for informational (Harsanyi) types. Mertens-Zamir and Brandenburger-Dekel define a type as an infinite hierarchy of beliefs over a set of possible parameters. Those parameters - or payoff types (Battigalli and Siniscalchi (2003)) - are by assumption exogenous and therefore require no further explanation. The interdependence of Harsanyi types arises from the interaction of the agents' beliefs. Agent 1's type influences agent 2's payoff because 1 has information about a payoff relevant parameter.

Interdependence in our setting is not related to a player's information. A player's personality specifies how the player reacts to the characteristics and personalities of other players and is independent of what the player knows. Our construction of preference types and the Mertens-Zamir and Brandenburger-Dekel construction of epistemic types 
are complementary. In a more general model, epistemic types (i.e., the infinite hierarchies of beliefs) can be defined given the interdependent preference types (i.e., the set of parameters).

In the concluding section, we discuss the relationship between our results and technically related issues in that literature. In particular, we discuss Bergemann and Morris (2009) and the literature on communication and consensus (Geanakoplos and Polemarchakis (1982), Cave (1983), Bacharach (1985), Parikh and Krasucki (1990)). All proofs are in the appendix.

\section{Interdependent Preference Models}

For simplicity, we restrict attention to the two person/player setting. The two-person setting simplifies the notation and the extension to $n$ players is straightforward.

The environment consists of a set of social outcomes, A, a set of characteristics, $\Omega$, and a collection of preferences $\mathcal{R}$ on $A$. The social outcome could be the quantity of a public good together with a division of its cost between the two players. A characteristic might specify a player's occupation or education. We assume that $A$ and $\Omega$ are compact metric spaces and that $\mathcal{R}$ is a nonempty, compact set of continuous preference relations ${ }^{3}$ on $A$.

An IPM is a triple $M=(T, \gamma, \omega)$ where $T$ is the type space, $\gamma$ is a function that assigns a preference to each pair of types, and $\omega$ is a function that identifies the characteristic of each type.

Definition: Let $T$ be a compact metric space, $\gamma: T \times T \rightarrow \mathcal{R}$ and $\omega: T \rightarrow \Omega$ be continuous functions. Then, $M=(T, \gamma, \omega)$ is an interdependent preference model (IPM).

The definition of an IPM assumes $\gamma$ is the same for both players and, therefore, assumes a symmetry between the two players. We can easily extend the analysis to asymmetric situations at the cost of complicating the notation. An alternative (and simpler) way to incorporate asymmetry is to define $\gamma\left(t, t^{\prime}\right)$ as a pair of preferences, one for when the

\footnotetext{
3 A continuous preference relation on $A$ is a complete and transitive binary relation $R$ such that the sets $\{y \in A \mid y R x\},\{y \in A \mid x R y\}$ are closed subsets of $A$.
} 
decision maker is assigned the role of agent 1 and another for when he is assigned the role of agent 2. With this modification, our model can be applied to asymmetric situations.

The type profile $\left(t, t^{\prime}\right)$ implies the preference profile

$$
\Gamma\left(t, t^{\prime}\right):=\left(\gamma\left(t, t^{\prime}\right), \gamma\left(t^{\prime}, t\right)\right)
$$

Below, we sometimes refer to an $\operatorname{IPM}(T, \Gamma, \omega)$. In that case, it is understood that $\Gamma$ satisfies (1) for some $\gamma: T \times T \rightarrow \mathcal{R}$.

The function $\gamma(t, \cdot): T \rightarrow \mathcal{R}$ describes how the agent's preference changes as a function

of the opponent's type. Hence, $\gamma(t, \cdot)$ represents an agent's personality. Note, however, that the type space $T$ is not a primitive of the economic environment. Therefore, $\gamma(t, \cdot)$ cannot serve as a satisfactory definition of an agents' personality. To be meaningful, a personality must be expressed in terms of the primitives $(A, \Omega, \mathcal{R})$. Next, we describe how this can be done.

\subsection{A Game of Manners}

Two agents, their meals in front of them, must choose between a polite and cumbersome $(P)$ or an impolite and efficient $(E)$ way to eat. Their preferences are interdependent, as described by the following IPM. Let $k \geq 1$ and $T=\{1, \ldots, 2 k-1\}$. Each type's preference depends only on the type's own action, and we identify it with its top element $P$ or $E$. Choose $\epsilon$ such that $0<\epsilon<1 / 2 k$ and define personalities as follows:

$$
\gamma(i, j)= \begin{cases}P & \text { if } i+j+(i-j) \epsilon>2 k \\ E & \text { if } i+j+(i-j) \epsilon \leq 2 k\end{cases}
$$

Higher types have a more polite personality and a more polite opponent makes a player more inclined to eat politely. Specifically, type 1 always prefers eating efficiently; type $2 k-1$ always prefers eating politely. More generally, all types $i>k$ prefer eating politely if and only if their opponent's type is greater or equal to $2 k-i$ while all types $i \leq k$ prefer eating politely if and only if their opponent's type is strictly greater than $2 k-i$. All types have the same characteristic; that is $\Omega$ is an arbitrary singleton set and hence, $\omega: T \rightarrow \Omega$ is the constant function.

The two agents play a waiting game in which they must decide when and how to eat. Time is discrete and indexed by $s=1,2, \ldots$ In each period, players simultaneously 
choose either $P, E$ or wait $(W)$. If both players choose $P$ or $E$ the game ends. If one player chooses $P$ or $E$, his opponent must choose $P$ or $E$ in the next period and the game ends.

Assume that, whenever a player has a unique preference for all possible types of his opponent, the player eats according to his preference. Otherwise, the player waits. ${ }^{4}$ This leads to the following sequence of play: In period 1, type 1 eats efficiently, type $2 k-1$ eats politely and all other types wait. Types 1 and $2 k-1$ have a preference that is independent of their opponents' type and therefore those types eat in the first period. If player 1 chooses $P$ or $E$ in period 1 and player 2 chooses $W$, then player 2 matches 1 's eating style in period 2. This follows since, in that case, player 2's type is in $\{2, \ldots, 2 k-2\}$ and, therefore, he prefers eating politely if his opponent is of type $2 k-1$ and efficiently if his opponent is of type 1 . If both players wait in period 1 , then the period 1 pattern is repeated in period 2 with types 2 and $2 k-2$ playing the role of types 1 and $2 k$. More generally, if both players wait in period $1, \ldots, s-1$, for $s \leq k-1$, then, in period $s$, type $s$ eats efficiently, type $2 k-s$ eats politely and all other players wait. If only one player eats in period $s$, his opponent matches his eating style in the following period. The game ends in at most $k$ periods.

The players' actions in this game can be interpreted as hierarchical preference statements. These statements will serve as a template for the construction of the type space in the next subsection.

Consider the actions in period 1 . Types who eat in period 1 have a preference that is independent of the opponent's type. Type 1 prefers eating efficiently irrespective of the opponent type; type $2 k-1$ prefers eating politely irrespective of the opponent type. Types who wait (all types other than 1 and $2 k-1$ ) have both $P$ and $E$ as possible preferences. Those types need to know more about the opponent before they can eat. Thus, period 1 play reveals - for each type - the set of possible eating preferences when nothing is known about the opponent. For type 1 this is $f_{1}=\{E\}$, for types $2, \ldots 2 k-2$ this is $f_{1}^{\prime}=\{P, E\}$ and for type $2 k-1$ this is $f_{1}^{\prime \prime}=\{P\}$. Thus,

$$
\Theta_{1}=\left\{f_{1}, f_{1}^{\prime}, f_{1}^{\prime \prime}\right\}
$$

is the set of preference statements conveyed by the actions in the first period.

\footnotetext{
4 It is straightforward to define an information structure and payoffs for the game such that the indicated behavior is the unique Nash equilibrium.
} 
Interpreted as a hierarchical preference statement, the period 2 action reveals a map that associates to each set of preferences (revealed in period 1) a set of possible preferences. For types 1 and $2 k-1$, there is nothing more to reveal. We therefore describe type 1 by the constant function $f_{2}^{E}\left(\theta_{1}\right)=\{E\}$ for all $\theta_{1} \in \Theta_{1}$ and type $2 k-1$ by the constant function $f_{2}^{P}\left(\theta_{1}\right)=\{P\}$ for all $\theta_{1} \in \Theta_{1}$. Types 2 and $2 k-2$ eat in period 2 and, therefore, for each period 1 revelation $\left(f_{1}, f_{1}^{\prime}\right.$ or $\left.f_{1}^{\prime \prime}\right)$, these types have a unique preference. For type 2 this corresponds to $f_{2}$ where

$$
f_{2}\left(f_{1}\right)=\{E\} ; f_{2}\left(f_{1}^{\prime}\right)=\{E\} ; f_{2}\left(f_{1}^{\prime \prime}\right)=\{P\}
$$

and for type $2 k-2$ this corresponds to $f_{2}^{\prime \prime}$ where

$$
f_{2}^{\prime \prime}\left(f_{1}\right)=\{E\} ; f_{2}^{\prime \prime}\left(f_{1}^{\prime}\right)=\{P\} ; f_{2}^{\prime \prime}\left(f_{1}^{\prime \prime}\right)=\{P\}
$$

Types $3, \ldots, 2 k-3$ wait also in period 2 if the opponent waited in period 1 because $P$ and $E$ remain possible preferences. This corresponds to $f_{2}^{\prime}$ where

$$
f_{2}^{\prime}\left(f_{1}\right)=\{E\} ; f_{2}^{\prime}\left(f_{1}^{\prime}\right)=\{P, E\} ; f_{2}^{\prime}\left(f_{1}^{\prime \prime}\right)=\{P\}
$$

Thus, interpreted as preference statements, the actions in the first two periods reveal an element of $\Theta_{2}$ where

$$
\Theta_{2}=\left\{\left(f_{1}, f_{2}^{E}\right),\left(f_{1}^{\prime}, f_{2}\right),\left(f_{1}^{\prime}, f_{2}^{\prime}\right),\left(f_{1}^{\prime}, f_{2}^{\prime \prime}\right),\left(f_{1}^{\prime \prime}, f_{2}^{P}\right)\right\}
$$

Proceeding recursively, the equilibrium actions in subsequent periods $s=3, \ldots, k$ can be translated into analogous preference statements. Each type then can be identified by a sequence of functions $\left\{f_{s}\right\}$ corresponding to a collection of hierarchical preference statements. The following subsection mimics this example to formulate a general hierarchical definition of types.

\section{$2.2 \quad$ Preference Response Hierarchies}

The hierarchy constructed in the game of manners is simpler than the general construction in two respects. First, the general model allows for multiple characteristics. 
Second, the hierarchy allows for types that distinguish themselves not just by their own preferences but also by how others react to them. To allow for this, the hierarchical preference statements involve sets of preference profiles rather than sets of preferences as in the game of manners.

Let $\mathcal{H}$ denote the collection of all closed subsets of preference profiles (closed subsets of $\mathcal{R} \times \mathcal{R}) .{ }^{5}$ For the compact metric space $X$, let $\mathcal{C}(X, \mathcal{H})$ denote the set of all functions $f: X \rightarrow \mathcal{H}$ that have a closed graph. ${ }^{6}$

Definition: A collection of nonempty compact sets $\left(\Theta_{0}, \Theta_{1}, \ldots\right)$ is a system of preference response hierarchies if $\Theta_{0}=\Omega$ and $\Theta_{n} \subset \Theta_{n-1} \times \mathcal{C}\left(\Theta_{n-1}, \mathcal{H}\right)$ for all $n \geq 1$.

The set $\Theta_{0}$ are the characteristics. An element $\theta_{1}=\left(\theta_{0}, f_{1}\right) \in \Theta_{1}$ consists of the person's own characteristic $\theta_{0}$ and a function $f_{1}: \Theta_{0} \rightarrow \mathcal{H}$ that associates to each opponent characteristic a set of preference profiles. This set has the following interpretation: $\left(R, R^{\prime}\right) \in f_{1}\left(\theta_{0}^{\prime}\right)$ if there is an opponent type with characteristic $\theta_{0}^{\prime}$ such that the player described by $\left(\theta_{0}, f_{1}\right)$ has preference $R$ and the opponent has preference $R^{\prime}$. Similarly, an element $\theta_{k} \in \Theta_{k}$ consists of the entry $\theta_{k-1}$ and the function $f_{k}: \Theta_{k-1} \rightarrow \mathcal{H}$ that specifies for each $\theta_{k-1}^{\prime}$ (of the opponent) the player's possible preference profiles.

\subsection{Consistent Preference Response Hierarchies}

Next, we impose two consistency conditions on the system of preference response hierarchies $\left(\Theta_{0}, \Theta_{1}, \ldots\right)$. To illustrate the first condition, let $\theta_{2}=\left(\theta_{0}, f_{1}, f_{2}\right) \in \Theta_{2}$. Then, $f_{1}\left(\bar{\theta}_{0}\right)$ is the set of possible preference profiles when the opponent's characteristic is $\bar{\theta}_{0}$ while $f_{2}\left(f_{1}^{\prime}, \bar{\theta}_{0}\right)$ is the set of possible preference profiles when the opponent has the partial hierarchy $\left(\bar{\theta}_{0}, f_{1}^{\prime}\right)$. If we fix $\bar{\theta}_{0}$ and form the union $\bigcup_{\left\{f_{1}^{\prime}:\left(f_{1}^{\prime}, \bar{\theta}_{0}\right) \in \Theta_{1}\right\}} f_{2}\left(f_{1}^{\prime}, \bar{\theta}_{0}\right)$, we obtain an alternative description of the set of possible preference profiles when the opponent's characteristic is $\bar{\theta}_{0}$. To be consistent, those two descriptions must lead to the same set of preferences:

$$
\bigcup_{\left\{f_{1}^{\prime}:\left(f_{1}^{\prime}, \bar{\theta}_{0}\right) \in \Theta_{1}\right\}} f_{2}\left(f_{1}^{\prime}, \bar{\theta}_{0}\right)=f_{1}\left(\bar{\theta}_{0}\right)
$$

5 We endow $\mathcal{H}$ with the Hausdorff topology. In the following, if $X_{j}$ is a metric space for all $j$ in some countable or finite index set $J$, we endow $\times_{j \in J} X_{j}$ with the sup metric.

6 Hence, $\mathcal{C}(X, \mathcal{H})$ is the set of upper hemi-continuous correspondences from $X$ to $\mathcal{R} \times \mathcal{R}$. 
Consider two partial hierarchies $\theta_{1}=\left(\theta_{0}, f_{1}\right), \theta_{1}^{\prime}=\left(\theta_{0}^{\prime}, f_{1}^{\prime}\right) \in \Theta_{1}$. The second consistency condition requires that the pair $\theta_{1}$ and $\theta_{1}^{\prime}$ be mutually consistent, that is, if $\theta_{1}$ describes player 1 and $\theta_{1}^{\prime}$ describe player 2 , then it must be possible that $f_{1}\left(\theta_{0}^{\prime}\right)$ and $f_{1}^{\prime}\left(\theta_{0}\right)$ are both correct. Put differently, there must be exist a preference profile $\left(R, R^{\prime}\right)$ such that

$$
\left(R, R^{\prime}\right) \in f_{1}\left(\theta_{0}^{\prime}\right) \text { and }\left(R^{\prime}, R\right) \in f_{1}^{\prime}\left(\theta_{0}\right)
$$

To illustrate condition $\left(C 2^{\prime}\right)$ suppose there are two alternatives $a, b$ and a partial hierarchy $\left(\theta_{0}, f_{1}\right)$ such that $f_{1}\left(\theta_{0}^{\prime}\right)=\{(a \succ b, a \succ b)\}$. This means that, for any possible opponent with characteristic $\theta_{0}^{\prime}$, both this player and his opponent prefer $a$ to $b$. Similarly, $f_{1}^{\prime}\left(\theta_{0}\right)=\{(b \succ a, b \succ a)\}$ means that, for any possible opponent with characteristic $\theta_{0}$, both this player and his opponent prefer $b$ to $a$. If $\left(\theta_{0}, f_{1}\right) \in \Theta_{1}$, then consistency requires that $\left(\theta_{0}^{\prime}, f_{1}^{\prime}\right) \notin \Theta_{1}$; otherwise, when one player has the partial hierarchy $\left(\theta_{0}, f_{1}\right)$ and the other has the partial hierarchy $\left(\theta_{0}^{\prime}, f_{1}^{\prime}\right)$, there is no preference profile consistent with both.

The following definition extends $\left(C 1^{\prime}\right)$ and $\left(C 2^{\prime}\right)$ to all levels of the hierarchy:

Definition: The system of preference response hierarchies $\left(\Theta_{0}, \Theta_{1}, \ldots\right)$ is consistent if and only if for all $n \geq 1$,

(i) for all $\left(\theta_{n-1}, f_{n}, f_{n+1}\right) \in \Theta_{n+1}$, and for all $\bar{\theta}_{n-1} \in \Theta_{n-1}$,

$$
f_{n}\left(\bar{\theta}_{n-1}\right)=\bigcup_{\left\{f_{n}^{\prime} \mid\left(\bar{\theta}_{n-1}, f_{n}^{\prime}\right) \in \Theta_{n}\right\}} f_{n+1}\left(\bar{\theta}_{n-1}, f_{n}^{\prime}\right)
$$

(ii) for all $\left(\theta_{n-1}, f_{n}\right),\left(\theta_{n-1}^{\prime}, f_{n}^{\prime}\right) \in \Theta_{n}$, there is $\left(R, R^{\prime}\right) \in \mathcal{R} \times \mathcal{R}$ such that

$$
\left(R, R^{\prime}\right) \in f_{n}\left(\theta_{n-1}^{\prime}\right) \text { and }\left(R^{\prime}, R\right) \in f_{n}^{\prime}\left(\theta_{n-1}\right)
$$

Henceforth, we refer to a system of consistent preference response hierarchies simply as a consistent hierarchy. 


\subsection{The Canonical Type Space}

Given a consistent hierarchy $\left(\Theta_{0}, \Theta_{1}, \ldots\right)$, a type is a sequence $\theta=\left(\theta_{0}, f_{1}, \ldots\right)$ such that, for all $n$, the $n$-truncation $\left(\theta_{0}, f_{1}, \ldots, f_{n}\right)$ of $\theta$ is an element of $\Theta_{n}$. Let

$$
\Theta^{*}:=\left\{\left(\theta_{0}, f_{1}, \ldots, f_{n}, \ldots\right) \in \Theta_{0} \times \prod_{n=1}^{\infty} \mathcal{C}\left(\Theta_{n-1}, \mathcal{H}\right) \mid\left(\theta_{0}, f_{1}, \ldots, f_{n}\right) \in \Theta_{n}, \forall n\right\}
$$

be the set of types for the consistent hierarchy $\left(\Theta_{0}, \Theta_{1}, \ldots\right)$. A set $\Theta \subset \Theta^{*}$ is a component of the canonical type space if $\Theta$ is compact and if, for every $\theta=\left(\theta_{0}, f_{1}, \ldots\right), \theta^{\prime}=\left(\theta_{0}^{\prime}, f_{1}^{\prime}, \ldots\right) \in$ $\Theta$

$$
\Psi\left(\theta, \theta^{\prime}\right):=\bigcap_{n \geq 0} f_{n+1}\left(\theta_{0}^{\prime}, f_{1}^{\prime}, \ldots, f_{n-1}^{\prime}\right) \text { is a singleton }
$$

The canonical type space $\mathcal{I}$ is the union of all components. For any $\Theta \in \mathcal{I}$, let $\Psi: \Theta \times \Theta \rightarrow \mathcal{R} \times \mathcal{R}$ denote the function that specifies a preference profile when the player is type $\theta$ and the opponent is type $\theta^{\prime}$. The function $\Psi(\theta, \cdot)$ is the personality of type $\theta$. It describes how the player responds to different opponent personalities. Requirement (C2) in the definition of consistency ensures that the function $\Psi$ satisfies the following symmetry condition:

$$
\Psi\left(\theta, \theta^{\prime}\right)=\left(R, R^{\prime}\right) \text { implies } \Psi\left(\theta^{\prime}, \theta\right)=\left(R^{\prime}, R\right)
$$

If $\Psi$ satisfies (S), we call it symmetric. We define $\phi: \Theta \rightarrow \Omega \times \mathcal{C}(\Theta, \mathcal{H})$ as the function that specifies, for every type $\theta \in \Theta$, the characteristic of $\theta$ and the mapping $\theta$ uses to assign preference profiles to opponent types. Hence,

$$
\phi(\theta):=\left(\theta_{0}, \Psi(\theta, \cdot)\right)
$$

Theorem 1: The function $\Psi$ is continuous and symmetric and $\phi$ is a homeomorphism from $\Theta$ to $\phi(\Theta)$.

It follows from Theorem 1 that any component $\Theta \in \mathcal{I}$ is an IPM: for a symmetric $\Psi$, there is a $\psi: \Theta \times \Theta \rightarrow \mathcal{R}$ such that $\Psi\left(\theta, \theta^{\prime}\right)=\left(\psi\left(\theta, \theta^{\prime}\right), \psi\left(\theta^{\prime}, \theta\right)\right)$. Then, since $\Theta$ is compact (by definition) and $\psi$ is continuous (by Theorem 1), it follows that every component of the 
canonical type space is an IPM. We record this observation as a corollary. Let $\omega(\theta)=\theta_{0}$ for all $\theta=\left(\theta_{0}, \ldots\right) \in \Theta$.

Corollary: If $\Theta \in \mathcal{I}$, then $(\Theta, \psi, \omega)$ is an IPM.

As we noted in section 1.5, our canonical type space provides a foundation for IPMs that is analogous to the Mertens and Zamir (1985)/Brandenburger and Dekel (1993) foundations for informational (Harsanyi) types. The difference is that interdependence in our setting is not related to a player's information. Rather, it describes how one player reacts to the characteristics and personalities of other players. In a more general model, epistemic types (i.e., the infinite hierarchies of beliefs) could be defined over interdependent preference types (i.e., the set of parameters). ${ }^{7}$

\section{Valid Models}

Suppose the environment has a single characteristic and two possible preferences, $a$ and $b$. Consider the following IPM:

$$
\begin{array}{ccc} 
& 1 & 2 \\
1 & a, a & b, b \\
2 & b, b & a, a
\end{array}
$$

Table 3

This IPM is not an element of the canonical type space. To see why not, note that all types have the same characteristic, there is nothing to report in round 0 . The round 1 set of possible preference profiles is $\{(a, a),(b, b)\}$ for both players. Since round 1 statements are identical for both types, all higher round statements must be identical as well. Hence, for all $n$ the function $f_{n}$ is constant (with value $\left.\{(a, a),(b, b)\}\right)$. The two types in this IPM have identical preference response hierarchies.

The IPM in table 3 illustrates a case in which the IPM introduces a distinction between types 1 and 2 that has no counterpart in terms of the model's primitives. Every preference statement that holds for type 1 is also true for type 2. Therefore, we cannot express the personalities of those two types as preference statements.

\footnotetext{
7 For example, a full description of the game of manners would describe the infinite hierarchy of beliefs of both players given the specified preference types.
} 
We can interpret the IPM in table 3 as an incomplete model. For example, it might be that the model omits a type.

$\begin{array}{cccc} & 1 & 2 & 3 \\ 1 & a, a & b, b & b, a \\ 2 & b, b & a, a & a, a \\ 3 & a, b & a, a & a, a\end{array}$

Table 4

In the IPM in table 4 , type 3 always prefers $a$ irrespective of the opponent's type. Type 2 accommodates this preference while type 1 does not. Hence, types 1 and 2 have different personalities, and can be distinguished through their response to type 3. The IPM depicted in table 3 could be interpreted as table 4 with an omitted type.

Alternatively, it might be that the model has omitted a characteristic: if types 1 and 2 have different characteristics (type 1 is wealthy, type 2 is poor) then the IPM in table 3 is a component of the canonical type space: both types have preference $a$ if the opponent's type has the same wealth and preference $b$ otherwise.

The two interpretations have different implications for applications. If players' behavior depends on the opponent's wealth (an omitted characteristic) then the observability of wealth is a key determinant of outcomes. If players' behavior depends on their response to some third personality type (type 3 ) then observability of wealth should have no effect, and instead the observability of past play will affect outcomes. Since the IPM is not a component of the canonical type space, we cannot express personalities in terms of the underlying primitives of the model. As a result, we cannot determine how information about the underlying primitives affects behavior.

Next, we provide a criterion (validity) that identifies whether or not an IPM is a component of the canonical type space. A partition $\mathcal{D}$ of $T$ is a pairwise disjoint collection of non-empty subsets such that $\bigcup_{D \in \mathcal{D}} D=T$. Let $D^{t}$ denote the unique element of $\mathcal{D}$ that contains $t$. The partition $\mathcal{D}=\{\{t\} \mid t \in T\}$ is called the finest partition. Let $(T, \gamma, \omega)$ be an IPM. Recall that

$$
\Gamma\left(t, t^{\prime}\right):=\left(\gamma\left(t, t^{\prime}\right), \gamma\left(t^{\prime}, t\right)\right)
$$

and define $\Gamma(t, D):=\left\{\Gamma\left(t, t^{\prime}\right) \mid t^{\prime} \in D\right\}$. 
Definition: The $\operatorname{IPM}(T, \gamma, \omega)$ is valid if the finest partition of $T$ is the only partition $\mathcal{D}$ such that $t^{\prime} \in D^{t} \in \mathcal{D}$ implies $\omega(t)=\omega\left(t^{\prime}\right)$ and $\Gamma(t, D)=\Gamma\left(t^{\prime}, D\right)$ for all $D \in \mathcal{D}$.

Validity requires that it be impossible to partition the type space in a manner that yields a partition element with multiple (indistinguishable) types. Theorem 2 shows that any valid IPM corresponds to a component $\Theta \in \mathcal{I}$. Two IPM's $(T, \gamma, \omega),\left(T^{\prime}, \gamma^{\prime}, \omega^{\prime}\right)$ are isomorphic if there exists a homeomorphism $\iota: T \rightarrow T^{\prime}$ such that $\omega(t)=\omega^{\prime}(\iota(t))$ and $\gamma(s, t)=\gamma^{\prime}(\iota(s), \iota(t))$ for all $s, t \in T$.

Theorem 2: An IPM is valid if and only if it is isomorphic to a component of the canonical type space.

We have interpreted the invalid IPM above as an incomplete model, either missing types or missing characteristics. Note that any finite invalid IPM can be "validated" by adding new types or new characteristics. Any model is obviously valid if each type has a distinct characteristic. Hence, validating an invalid IPM by assigning distinct characteristics to each type is straightforward. For the missing types interpretation, it is easy to show that any finite, invalid IPM can be embedded in a valid IPM with a larger type space. More precisely, assume that $\mathcal{R}$ contains at least 2 preferences and consider a finite $\operatorname{IPM}(T, \gamma, \omega)$. Then, there exists a valid IPM $(\hat{T}, \hat{\gamma}, \hat{\omega})$ such that $T \subset \hat{T}, \gamma(t)=\hat{\gamma}(t)$ for all $t \in T$ and $\omega(t)=\hat{\omega}(t)$ for all $t \in T$.

\section{Two Consequences of Validity}

We relate validity to two objectives of a model of interdependent preferences. The first objective is to answer the following question: under what conditions can an outside observer identify the types of a collection of interacting economic agents? Specifically, we consider a population of agents who know each other's types, are matched into pairs and then take economic actions that reveal their preferences. The observer sees the preferences of a sample of agents. We show that if the sample is rich (in a sense defined below), then the observer can identify the types of a valid model. Conversely, rich samples cannot identify all types of an invalid model. Thus, validity is equivalent to the observability of types. 
As in the game of manners, consider a setting in which players have a common interest to communicate each other's type. Our second objective is to answer the following question: under what conditions can such a communication be successful? We require that players communicate their types with a language based on economic primitives. Thus, players can refer to their characteristics and preferences but cannot refer to their types directly. The preference response hierarchies, defined above, are a particular example of such a communication. Hence, Theorem 2 shows that in a valid model, players can communicate their types. Below, we prove a converse. If the IPM is not valid then players cannot communicate their types in a language based on economic primitives. Thus, validity is necessary and sufficient for types to be communicable.

\subsection{Validity and Observability}

Consider a setting in which players from a population are repeatedly matched. An underlying IPM determines players' preferences in each match. Types are common knowledge among the players and, therefore, players have no incentive to conceal their preferences. Each pair of matched players make a series of choices that reveal the preferences of both players.

In this section, we ask the following question: suppose an observer does not know the players' types but observes a sample of matches and, by observing the corresponding choices, learns the preferences of the players in those matches. Can this observer deduce a particular player's type with a sufficiently rich sample of matches? We provide a formal definition of a rich sample for a particular player and show that if the underlying IPM is valid, then every rich sample for player $i$ will enable the observer to deduce $i$ 's type. Conversely, if the IPM is not valid, we can always find a rich sample for some player $i$ that does not reveal player $i$ 's type.

Let $(T, \gamma)$ be an IPM with a finite $k$ types. To simplify the notation, we assume that all types have the same characteristic. A sample for this IPM is a finite set of players $N=\{1, \ldots, n\}$, an assignment of types $t_{i} \in T$ for the players $i \in N$ and a set $\Sigma \subset N \times N$ of interactions. Thus, $i j \in \Sigma$ means that the sample contains an observation in which player $i$ was matched with player $j$. This interaction enables the observer to learn the preferences of both $i$ and $j$ when confronting each other. Since each match reveals the preferences of 
both players, we assume, without loss of generality, that two players interact at most once and we do not distinguish between the interaction $i j$ and $j i$; that is, $i j \in \Sigma$ implies $j i \in \Sigma$.

Given the sample $\Sigma$, the observer learns $\Gamma\left(t_{i}, t_{j}\right)$ for all $i j \in \Sigma$ but not the type $t_{i}$ of player $i$. We call a function $\tau: N \rightarrow T$ a prediction of players' types. The prediction $\tau$ is consistent with the sample $\Sigma$ if $\Gamma(\tau(i), \tau(j))=\Gamma\left(t_{i}, t_{j}\right)$ for all $i j \in \Sigma$. The sample $\Sigma$ reveals the type of player $i$ if $\tau(i)=\tau^{\prime}(i)$ for all $\tau, \tau^{\prime}$ consistent with $\Sigma$. Hence, a sample reveals the type of player $i$ if and only if $\tau(i)=t_{i}$; that is, if and only if it enables the observer to learn the type of player $i$.

A sample contains a full set of interactions for player $i$ if $i$ interacts with at least one player of every type.

Definition: The sample $\Sigma$ contains a full set of interactions for player $i$ if for all $t \in T$ there is $i j \in \Sigma$ with $t_{j}=t$.

We say that a sample is $i$-complete if it contains a full set of interactions for $i$, for $i$ 's opponents, of $i$ 's opponents' opponents, and so on, for $k$ rounds. The number $k$ is the number of types in the underlying IPM. To deduce player $i$ 's type, the observer requires a full set of interactions for player $i$. But, to understand these interactions, the observer requires a full set of interactions for $i$ 's opponents in those interactions and so on. Theorem 3 establishes that for an valid IPM, these requirements do not lead to an infinite regress; $k$-rounds is enough.

Definition: $\quad \Sigma$ is $i$-complete if (i) $\Sigma$ contains a full set of interactions for $i$ and (ii) $\Sigma$ contains a full set of interactions for all $j$ such that $\left\{i j_{1}, j_{1} j_{2}, \ldots, j_{r} j\right\} \subset \Sigma$ for some $1 \leq r \leq k$

We say that the types of the IPM are observable if, every $i$-complete sample for the IPM reveals the type of player $i$.

Theorem 3: The types of the IPM $(T, \gamma)$ are observable if and only if $(T, \gamma)$ is valid.

Theorem 3 shows that validity is closely related to the empirical identifiability of types. Given enough data, an outside observer can determine the players' types if the underlying 
IPM is valid. Thus, after observing a sample that is both $i$-complete and $j$-complete, we can make an out-of-sample prediction about behavior in an $i j$ interaction provided that the model is valid. In contrast, an invalid model has types that will make identical choices even in a complete data set often rendering out-of-sample predictions impossible.

\subsection{Validity and Communicability}

We have interpreted preference response hierarchies as players' conditional preference statements that gradually reveal their types. Those hierarchies impose a particular protocol of how these statements unfold. In this section, we show that no other protocol can do better. More formally, we introduce a general framework for communication and show that agents can reveal their types through communication if and only if the IPM is valid. Hence, types are communicable if and only if the IPM is a component of the canonical type space.

For simplicity, we assume that there is a single characteristic ${ }^{8}$ and a finite number of behavioral types. To formulate a model of communication, we need to translate the IPM into an epistemic model. An epistemic model is a finite set of states $S$ and a pair of partitions $\mathcal{T}_{1}$ and $\mathcal{T}_{2}$ of $S$ that represent the players' knowledge. Elements of $\mathcal{T}_{i}$ are player $i$ 's epistemic types. Let $(T, \gamma)$ be an IPM with a single characteristic and a finite set of types. In the equivalent epistemic model, each player knows his own type and knows nothing about his opponent's type; that is, any type in $T$ is possible. We also assume that the opponent's type is the only relevant information that players lack. Hence, we identify $S$ with $T \times T, \mathcal{T}_{1}$ with $\{\{t\} \times T \mid t \in T\}$ and $\mathcal{T}_{2}$ with $\{T \times\{t\} \mid t \in T\}$.

A collection of subsets $\mathcal{L}$ of a set $S$ is an algebra (or equivalently, a language) if it contains $S$ and is closed under unions and complements. An element of the language is a word. The primitives of an interactive preference model are preference statements. Hence, the relevant language is the the language of preferences. ${ }^{9} \mathrm{~A}$ subset $B$ of $S$ is a word in the language of preferences if $B=\Gamma^{-1}(V)$ for some set of preference profiles $V \in \mathcal{H}$. The language of preferences consists of all the words of this form.

\footnotetext{
8 Extending the analysis below to IPMs with multiple characteristics is straightforward. We assume a single characteristic to keep the notation simple.

9 The formalism developed here can be used to define communicability with respect to any given language.
} 
A person with knowledge $\mathcal{T}_{i}$ can convey information to his opponent using the word $B$ with one of the following two statements: "I know B" or "I don't know not B." These statements enable the player's opponent to deduce one of the following two facts about the player's epistemic type $A$ : $A \subset B$ or $A \cap(S \backslash B) \neq \emptyset$. The knowledge that each player can convey to his opponent in one round of communication in language $\mathcal{L}$ is the sum of all such statements for every $B \in \mathcal{L}$.

After an initial round of information exchange, both players will be able to make additional statements given what they have learned in the first round. An exchange of such statements will again lead to more knowledge and so on. After finitely many rounds this process will reach a fixed point beyond which further knowledge exchange is impossible. This ultimate level of knowledge identifies all that is communicable in the language of preferences and if this knowledge includes knowledge of the opponent's type, we say that the IPM in epistemic form is communicable in the language of preferences. For a precise definition of communicability, see Appendix D.

Theorem 4: A finite IPM in epistemic form is communicable in the language of preferences if and only if it is equivalent to a valid IPM.

We interpret communicability as a test that a well-defined, self-contained model of interdependent preference types must satisfy: responding to the opponent's personality requires understanding his personality and each player's understanding is the sum of what he knows at the outset (i.e., his type) and what he can learn through communication with the other player. ${ }^{10} \mathrm{~A}$ model that violates this communicability test can, at best, be interpreted as an incomplete model: players respond to personality types that are well defined in a larger context (in a richer model) but ill-defined in the IPM at hand.

\footnotetext{
10 Since we wish to identify what can be understood in principle, we are ignoring incentives. In practice, an agent may learn less than what can be communicated in the language of preferences since his opponent may strategically withhold information.
} 


\section{Reciprocity}

One preference is kinder than another if it places more value on the well-being of the opponent. A nicer personality is one that is kinder to every personality type. Finally, a reciprocating personality ${ }^{11}$ is one that is kinder to nicer opponents. Hence, in our formal definition of reciprocity, we assume an exogenous "kindness" ranking on preferences. The literature often assumes that individuals have exogenously specified selfish utilities and identifies altruism (or generosity or kindness) with the relative weight a person puts on other's selfish utility. For example, let $(x, y) \in A=[0,100] \times[0,100]$ be the vector of consumptions and assume that selfish utilities are linear. Then, let

$$
U^{r}(x, y)=x+r y
$$

be the utility function representing the preference with parameter $r \in[-1,1]$. A natural kindness order on these preferences is the " $\geq$ " ranking of their parameters. That is, the preference (with parameter) $r$ is kinder than the preference $r^{\prime}$ if and only if $r \geq r^{\prime}$.

More generally, we assume that there is a continuous one-to-one function $\tau: \mathcal{R} \rightarrow \mathbb{R}$ and interpret $\tau(R) \geq \tau\left(R^{\prime}\right)$ to mean $R$ is kinder than $R^{\prime}$. We say that $(T, \gamma)$ is an ordered IPM when such a $\tau$ exists. When the IPM is ordered, it is convenient to identify each preference $R$ with $\tau(R)$ and suppress preferences. Then, we let $\delta=\tau \circ \gamma$ and refer to $(T, \delta)$ as an ordered IPM.

Definition: In an ordered IPM, type $t$ is nicer than type $t^{\prime}$ if $\delta\left(t, t^{\prime \prime}\right) \geq \delta\left(t^{\prime}, t^{\prime \prime}\right)$ for all $t^{\prime \prime}$; type $t$ reciprocates if $\delta\left(t, t^{\prime}\right) \geq \delta\left(t, t^{\prime \prime}\right)$ whenever $t^{\prime}$ is nicer than $t^{\prime \prime}$. An ordered IPM is a reciprocity model if it is valid, the niceness relation is complete and every type reciprocates.

The simplest kind of an ordered IPM is one in which types are also real numbers. In such a model, if $\delta$ is increasing in the first argument, then bigger types are nicer. If it is also increasing in the second argument, then all types reciprocate. Theorem 4 shows that adding a mild genericity condition to such a model ensures validity. The theorem also shows that, in fact, all reciprocity models are of this kind.

\footnotetext{
11 For simplicity, we assume throughout this section that all types have the same characteristic and hence use type and personality interchangeably.
} 
Definition: $\quad$ The ordered IPM $(T, \delta)$ is simple if $T \subset \mathbb{R}$; a simple IPM is increasing if $\delta$ is nondecreasing in both arguments and $\delta(t, \cdot)=\delta\left(t^{\prime}, \cdot\right), \delta(\cdot, t)=\delta\left(\cdot, t^{\prime}\right)$ implies $t=t^{\prime}$.

Note that simple IPMs can accommodate both positive and negative reciprocity. ${ }^{12}$ Let $r^{*}$ be a reference level of fairness and let

$$
\delta(t, e(t))=r^{*}
$$

Type $t$ 's opponent exceeds the reference level of fairness whenever $t^{\prime} \geq e(t)$. So, type $t$ exhibits positive reciprocity if $\delta(t, \cdot)$ is flat or nearly flat when $t^{\prime}<e(t)$ but steep when $t^{\prime}>e(t)$. Thus, type $t$ reciprocates when opponent types are nicer than the threshold $e(t)$ but does not reciprocate when opponent types are below $e(t)$. Conversely, type $t$ exhibits negative reciprocity if $\delta(t, \cdot)$ is steep when $t^{\prime}<e(t)$ but flat when $t^{\prime}>e(t)$. Thus, type $t$ reciprocates when opponent types are less nice than the threshold $e(t)$ but does not reciprocate when opponent types are above $e(t)$.

Theorem 5: An ordered IPM is a reciprocity model if and only if it is isomorphic to some simple increasing IPM.

To prove Theorem 5, we first show that if $\delta$ is nondecreasing in both arguments, then the simple IPM $(T, \delta)$ is valid if and only if $\delta$ satisfies the genericity condition $\delta(t, \cdot)=\delta\left(t^{\prime}, \cdot\right)$ and $\delta(\cdot, t)=\delta\left(\cdot, t^{\prime}\right)$ implies $t=t^{\prime}$. Hence, nondecreasingness of $\delta$ ensures that each type in a simple model is reciprocating and, given nondecreasingness, the genericity condition is exactly what is needed for a simple model to be valid. For the converse, we show that validity together with the continuity and compactness properties of IPMs ensure that the types in a reciprocity model can be identified with real numbers.

Next, we examine the special case of an ordered IPM with two possible preference profiles. Hence, we identify $\mathcal{R}$ with $\{0,1\}$ where 1 is the generous (i.e., kinder) preference, 0 is the selfish preference and assume that $\delta\left(t, t^{\prime}\right)=\delta\left(t^{\prime}, t\right)=0$ or $\delta\left(t, t^{\prime}\right)=\delta\left(t^{\prime}, t\right)=1$ for all $t, t^{\prime} \in T$. We call such models binary IPMs.

As we show in Theorem 6 below, there are only two classes of valid binary IPMs. Both classes consist of simple increasing IPMs and hence are reciprocity models. In the first

\footnotetext{
12 Dohmen, Falk, Huffman and Sunde (2009) show that positive reciprocating people (i.e., types) enjoy better job market outcomes and more life satisfaction than negative reciprocating types.
} 
class, the highest type is generous irrespective of the opponent's type and all opponents are generous when matched with the highest type. Let

$$
\delta_{m}(i, j)= \begin{cases}1 & \text { if } i+j>m \\ 0 & \text { otherwise }\end{cases}
$$

Let $K=\{1, \ldots, k\}$ be the set of types. Then, $M_{k}^{0}=\left(K, \delta_{k}\right)$ is the first class of binary IPMs. In the second class, the lowest type is always selfish irrespective of the opponent's type and all opponents are selfish when they are matched with the lowest type. This class of binary IPMs is $M_{k}^{1}=\left(K, \delta_{k+1}\right)$. Theorem 6 shows that these are the only valid binary IPMs.

Theorem 6: A binary IPM is valid if and only if it is isomorphic to some $M_{k}^{i}$.

It is easy to verify that every $M_{k}^{i}$ is a reciprocity model. Hence, Theorem 6 establishes that valid binary IPMs are reciprocity models. To gain intuition for Theorem 6 , first note that, by compactness and continuity, a binary reciprocity model must have a finite number of types $\{1, \ldots, m\}$. If $m=1$, there is nothing to prove. Suppose the result is true whenever $m=k$ and let $m=k+1$. Since there are only two preference profiles, validity ensures that $\delta(t, \cdot)$ is constant for some type $t$. Suppose this constant is 1 and without loss of generality let $t=k+1$. Then, we show that the validity of $(\{1, \ldots, k+1\}, \delta)$ implies that $(\{1, \ldots, k\}, \delta)$ is valid and that there exists no $t \leq k$ such that $\delta(t, \cdot)=1$. Then, by the inductive hypothesis, the restriction of $\delta$ to $\{1, \ldots, k\}$ must be $\delta_{k+1}$, implying that $\delta=\delta_{k+1}$.

\subsection{Modeling Intentions with IPMs}

As we noted in the introduction, IPMs are not adequate for modeling all departures from the standard framework. By the standard framework, we mean what Falk and Fischbacher (2006) call the "consequentialistic perspective," that is, any model in which the physical description of outcomes is sufficient for identifying the utility of outcomes. Even some forms of reciprocity may fall outside of the reach of IPMs. For example, a player may care only about whether or not another player acted generously and not about the other player's (persistent) personality. ${ }^{13}$

13 Falk and Fischbacher's (2006) definition of reciprocity reflects this view. 
However, when modeling intentions, identifying persistent attributes as the carriers of utility has some advantages. We discussed those advantages in the introduction: the implied separation of preferences from institutions facilitates the analysis of economic design problems. Here, we will discuss more closely the second advantage: it enables reciprocity models to differentiate between acting generously out of self-interest and genuine kindness.

Consider the following game: player 1 is either generous/trusts $(g)$ player 2 or he does not $(s)$. Afterwards, nature chooses either 0 or 1 . If nature chooses 0 , the game ends. The outcome $(g, 0)$ yields $(70,30)$; that is, 70 dollars for player 1 and 30 dollars for player 2 while $(s, 0)$ yields $(80,0)$. If nature chooses 1 , then player 2 chooses an action; she either accepts player 1's decision $(a)$ or declines it $(d)$. The outcome $(g, 1, a)$ yields the monetary payoffs $(70,30)$ while $(s, 1, a)$ yields the monetary payoffs $(80,0)$. If player 2 chooses $d$, she gets 40 dollars and player 1 gets 0 dollars. That is, $(g, 1, d)$ and $(s, 1, d)$ both yield the monetary payoffs $(0,40)$. Let $\alpha \in(0,1)$ be the probability that nature chooses 1 .

Consider how changing $\alpha$ might affect behavior. First, let $\alpha$ be close to 1 . Then, if player 1 chooses $s$ and nature chooses 1 , player 2 is likely to choose $d$ : by choosing $s$, player 1 has shown no generocity/trust and therefore player 2 is likely to be ungenerous as well. Knowing this, player 1 believes he is likely to get 0 if he chooses $s$. Hence, when $\alpha$ is high, player 1 will be inclined to choose $g$ even if he puts no weight on 2's well-being. Thus, player 1's generocity/trust in this case may be strategic.

In contrast, when $\alpha$ is close to zero, the action $g$ reveals a genuinely generous player 1; had he chosen $s$, he would have (almost) guaranteed himself 80 and he is giving this up for player 2's benefit. Therefore, conditional on the node $(g, 1)$ being reached, we would expect player 2 to be more inclined to honor player 1's trust when $\alpha$ is low than when it is high.

Thus, when $\alpha$ is close to $0, g$ is proof of player 1's good intentions and is fully rewarded. When $\alpha$ is close to $1, g$ can mean that player 1 has good intentions or that he would like player 2 to think he has good intentions. The possibility that player 1's generosity is not genuine should make player 2 less reciprocating. 
To see how a reciprocity model can match the intuition outlined above, we will model preferences with the binary IPM $M_{2}^{0}$ defined in the previous section. To be concrete, let $r \in\{0,1\}$ be the preference that $U^{r}$ below represents:

$$
U^{r}(x, y)=x+r y
$$

Then, consider the IPM $(\{1,2\}, \delta)$, where

$$
\delta\left(t, t^{\prime}\right)=1 \text { if and only if } t+t^{\prime}>2 \text {. }
$$

Assume that both players are drawn from the same population with $10 \%$ type 2's. For $\alpha$ sufficiently small, the game above has a unique equilibrium: player 1 chooses $g$ if and only if he is type 2 and player 2 always accepts $g$ and accepts $s$ if and only if she is type 2 . For $\alpha$ close to 1 , the game again has a unique equilibrium: player 1 chooses $g$ for sure if he is type 2 and randomizes between $g$ and $s$ if he is type 1 . Player 2 accepts either offer for sure if she is type 2 and, if she is type 1 , rejects $s$ for sure and randomizes between $a$ and $d$ against $g$.

These two equilibria match the intuition above exactly: if $\alpha$ is close to 0 , choosing $g$ is unambiguously generous and such generosity is rewarded. If $\alpha$ is close to 1 , both generosity and self-interest are possible motives for choosing $g$ and therefore player 2's response is more qualified; sometimes she reciprocates and sometimes she doesn't.

Stanca, Bruni and Corazzini (2007) run an experiment very similar to the game described above. In the experiment, a gift exchange game is repeated twice. In period 1 , player I gives to player II. In period 2, the game is repeated with the roles reversed. The experiment considers two treatments. In the first, subjects are not told that the game will be repeated whereas in the second treatment subjects are told the game will be repeated. In terms of the game described above, the two treatments corresponds to $\alpha=1$ and $\alpha=0 .{ }^{14}$ The experiment confirms the hypothesis that reciprocity is smaller when $\alpha=1$ than when $\alpha=0$. Stance et al. write, "not only do subjects reciprocate more in the NI-treatment on average, but also a larger fraction of the subjects do not display any

\footnotetext{
14 Stanca et al. use a GPS-based framework to analyze the game. In that framework, the player's utility function changes with the treatment to reflect less incentive to reciprocate when $\alpha$ is 1 .
} 
reciprocal behavior when strategic motivations cannot be ruled out. Strassmair (2009) performs a similar experiment but chooses the values $\alpha=1 / 2$ and $\alpha=.9$ in her treatment. ${ }^{15}$ Strassmair (2009) finds no effect of varying the parameter $\alpha$ on reciprocity.

\section{Related Literature on Belief (or Possibility) Hierarchies}

That each type can be identified with a unique hierarchy of preference statements is a central property of our model. The same objective - relating differences in types to differences in payoff relevant primitives - can also be pursued when types are exogenous parameters. ${ }^{16}$ Bergemann and Morris (2007), (2009) and Bergemann, Morris and Takahashi (2010) establish that this question plays a central role in implementation theory. Bergemann and Morris (2007) permit asymmetric information (i.e., players form conjectures over their opponents' preference types) and they prove two results in which conditions similar to validity play a role.

The first result shows that if a condition slightly stronger than validity is violated, then there are types that are strategically equivalent; that is, have the same rationalizable actions in every game. The second result presents a further strengthening of validity that is necessary and sufficient for two types to have disjoint sets of rationalizable actions in some game. Thus, Bergemann and Morris (2007) give conditions under which we can find a game that elicits payoff types uniformly over all belief types. We provide a more detailed discussion of the relationship in the supplementary appendix. There we also discuss in detail the relationship between our Theorem 4 and work on communication by Geanakoplos and Polemarchakis (1982), Cave (1983), Bacharach (1985) and Parkiks and Krasucki (1990). A key difference is that our model allows conditional statements such as "had you told me $x$, I would have said $y$ " whereas such statements are not possible in the CavesBacharach-Parikh and Krasucki models of communication protocols. Hence, our model of communication allows (weakly) more "knowledge sharing" than any communication protocol. Furthermore, there are examples in which our model allows strictly more knowledge sharing.

\footnotetext{
15 Similar to Stanca et al (2007), Strassmair (2009) employs a GPS-based model in which the utility function changes as a function of $\alpha$.

16 For example, Ely and Peski (2006) point out that in standard models of incomplete information, two different types may have exactly the same hierarchy of beliefs. See also Dekel, Fudenberg and Morris (2006a), (2006b) for related work. In an earlier version of this paper (Gul and Pesendorfer (2005)), we show that an IPM is valid if and only if each type is uniquely identified through its possibility hierarchy. The latter concept is due to Mariotti, Meier and Piccione (2005).
} 


\section{Appendix A: Notation and Definitions}

\subsection{Preliminaries}

We use the following notation and definitions. When $X_{j}$ is a metric space for all $j$ in some countable or finite index set $J$, we endow $\times_{j \in J} X_{j}$ with the sup metric. For any compact metric space $X$, let $\mathcal{H}_{X}$ be the set of all nonempty, closed subsets of $X$ and endow $\mathcal{H}_{X}$ with the Hausdorff topology. For the compact metric spaces $X, Z$ let $\mathcal{C}\left(X, \mathcal{H}_{Z}\right)$ denote the set of all functions $f: X \rightarrow \mathcal{H}_{Z}$ such that their graph $G(f)=\{(x, z) \in$ $X \times Z \mid z \in f(x)\}$ is closed in $X \times Z{ }^{17}$ We endow $\mathcal{C}\left(X, \mathcal{H}_{Z}\right)$ with the following metric: $d(f, g)=d_{H}(G(f), G(g))$, where $d_{H}$ is the Hausdorff metric on the set of all nonempty closed subsets of $X \times Z$. We identify the function $f: X \rightarrow Z$ with the function $\bar{f}: X \rightarrow \mathcal{H}_{Z}$ such that $\bar{f}(x)=\{f(x)\}$ for all $x \in X$. It is easy to verify that such a function $f$ is an element of $\mathcal{C}\left(X, \mathcal{H}_{Z}\right)$ if and only if $f$ is continuous. We use $\mathcal{C}(X, Z) \subset \mathcal{C}\left(X, \mathcal{H}_{Z}\right)$ to denote the set of continuous functions from $X$ to $Z$. We let $\mathcal{H}$ denote $\mathcal{H}_{\mathcal{R} \times \mathcal{R}}$, the collection of all closed subsets of preference profiles (closed subsets of $\mathcal{R} \times \mathcal{R}$ ).

\section{Appendix B: Proof of Theorems 1 and 2}

Let $Z$ be a compact metric space. For any sequence $A_{n} \in \mathcal{H}_{Z}$, let

$$
\begin{aligned}
& \underline{\lim A_{n}}=\left\{z \in Z \mid z=\lim z_{n} \text { for some sequence } z_{n} \text { such that } z_{n} \in A_{n} \text { for all } n\right\} \\
& \varlimsup \\
& \lim A_{n}=\left\{z \in Z \mid z=\lim z_{n_{j}} \text { for some sequence } z_{n_{j}} \text { such that } z_{n_{j}} \in A_{n_{j}} \text { for all } j\right\}
\end{aligned}
$$

Let $X$ be a metric space and $p: X \rightarrow \mathcal{H}_{Z}$. We say that $p$ is Hausdorff continuous if it is a continuous mapping from the metric space $X$ to the metric space $\mathcal{H}_{Z}$. Note that if $p$ is a Hausdorff continuous mapping from $X$ to $\mathcal{H}_{Z}$, then $p \in \mathcal{C}\left(X, \mathcal{H}_{Z}\right)$. However, the converse is not true.

Lemma 1: Let $X, Y, Y^{\prime}, Z$ be nonempty compact metric spaces, $q \in \mathcal{C}(X \times Y, Z), p \in$ $\mathcal{C}\left(Y^{\prime}, \mathcal{H}_{Y}\right)$, and $r \in \mathcal{C}\left(Y^{\prime}, Y\right)$. Then, (i) $A_{n} \in \mathcal{H}_{Z}$ converges to $A$ (in the Hausdorff topology) if and only if $\underline{\lim } A_{n}=\varlimsup \lim A_{n}=A$. (ii) $x_{n} \in X$ converges to $x$ implies $q\left(x_{n}, B\right)$ converges to $q(x, B)$ for all $B \in \mathcal{H}_{Y}$. (iii) If $q^{*}\left(x, y^{\prime}\right)=q\left(x, p\left(y^{\prime}\right)\right)$ for all $x \in X, y^{\prime} \in Y^{\prime}$ then $q^{*} \in \mathcal{C}\left(X \times Y^{\prime}, \mathcal{H}_{Z}\right)$. (iv) If $r$ is onto, then $r^{-1} \in \mathcal{C}\left(Y, \mathcal{H}_{Y^{\prime}}\right)$.

17 Hence, $\mathcal{C}\left(X, \mathcal{H}_{Z}\right)$ is the set of upper hemi-continuous correspondences from $X$ to $Z$. 
Proof: Part (i) is a standard result. See Brown and Pearcy (1995).

(ii) Suppose $x_{n} \in X$ converges to $x$. Let $z_{n_{j}} \in q\left(x_{n_{j}}, B\right)$ be such that $\lim z_{n_{j}}=z$. Hence, $z_{n_{j}}=q\left(x_{n_{j}}, y_{n_{j}}\right)$ for some $y_{n_{j}} \in B$. Since $B$ is compact, we can without loss of generality assume $y_{n_{j}}$ converges to some $y \in B$. Then, the continuity of $q$ ensures $z=$ $q(x, y)$ and therefore $z \in q(x, B)$ proving that $\varlimsup q\left(x_{n}, B\right) \subset q(x, B)$. If $z \in q(x, B)$, then there exists $y \in B$ such that $z=q(x, y)$. Since $q$ is continuous, we have $z=\lim q\left(x_{n}, y\right)$. Hence, $q(x, B) \subset \underline{\lim } q\left(x_{n}, B\right)$. Since, $\underline{\lim } q\left(x_{n}, B\right) \subset \varlimsup \lim q\left(x_{n}, B\right) \subset q(x, B)$, we conclude $\underline{\lim } q\left(x_{n}, B\right)=q(x, B)=\varlimsup \lim q\left(x_{n}, B\right)$ as desired.

(iii) Suppose $\left(x_{n}, y_{n}^{\prime}\right)$ converges to $\left(x, y^{\prime}\right)$ and $z_{n} \in q^{*}\left(x_{n}, y_{n}^{\prime}\right)$ converges to $z$. Pick $y_{n} \in p\left(y_{n}^{\prime}\right)$ such that $q\left(x_{n}, y_{n}\right)=z_{n}$. Since $Y$ is compact, we can assume that $y_{n}$ converges to some $y$. Since $p \in \mathcal{C}\left(Y^{\prime}, \mathcal{H}_{Y}\right)$, we conclude that $y \in p\left(y^{\prime}\right)$ and since $q$ is continuous, $q(x, y)=z$. Therefore, $z \in q^{*}\left(x, y^{\prime}\right)$, proving that $q^{*} \in \mathcal{C}\left(X \times Y, \mathcal{H}_{Z}\right)$.

(iv) The continuity and ontoness of $r$ ensures that $r^{-1}$ maps $Y$ into $\mathcal{H}_{Y^{\prime}}$. Assume that $y_{n}$ converges to $y, y_{n}^{\prime} \in r^{-1}\left(y_{n}\right)$ and $y_{n}^{\prime}$ converges to $y^{\prime}$. Then, $r\left(y_{n}^{\prime}\right)=y_{n}$ for all $n$ and by continuity $r\left(y^{\prime}\right)=y$. Therefore, $y^{\prime} \in r^{-1}(y)$ as desired.

Lemma 2: Let $X$ and $Z$ be compact metric spaces. Suppose $p_{n} \in \mathcal{C}\left(X, \mathcal{H}_{Z}\right)$ and $p_{n+1}(x) \subset p_{n}(x)$ for all $n \geq 1, x \in X$. Let $p(x):=\bigcap_{n \geq 1} p_{n}(x)$ and assume $p(x)$ is a singleton for all $x \in X$. Then, (i) $p$ is continuous and (ii) $p_{n}$ converges to $p$.

Proof: Obviously, $\bigcap_{n \geq 1} G\left(p_{n}\right)=G(p)$. Since $p_{n} \in \mathcal{C}\left(X, \mathcal{H}_{Z}\right)$ and $X, Z$ are compact, so is $G\left(p_{n}\right)$. Therefore $G(p)$ is compact (and therefore closed) as well. Since $p$ is a function and both $X, Z$ are compact, the fact that $p$ has a closed graph implies that $p$ is continuous.

To prove (ii), it is enough to show that if $G_{n}$ is a sequence of compact sets such that $G_{n+1} \subset G_{n}$, then $G_{n}$ converges (in the Hausdorff topology) to $G:=\bigcap_{n} G_{n}$. If not, since $G$ is compact, we could find $\epsilon>0$ and $y_{n} \in G_{n}$ converging to some $y \in G_{1}$ such that $y \notin G$. Hence, there is $k$ such that $y \notin G_{k}$. Since the $G_{k}$ is compact and $G_{n} \subset G_{k}$ for all $n>k$, there is $\epsilon>0$ such that $d\left(y^{\prime}, y\right) \geq \epsilon$ for all $y^{\prime} \in G_{n}$ and $n \geq k$, contradicting the fact that $y_{n} \in G_{n}$ for all $n$ and $\lim y_{n}=y$.

We say that $p_{n} \in \mathcal{C}\left(X, \mathcal{H}_{Z}\right)$ converges to $p \in \mathcal{C}\left(X, \mathcal{H}_{Z}\right)$ uniformly if for all $\epsilon>0$, there exists $N$ such that $n \geq N$ implies $d\left(p_{n}(x), p(x)\right)<\epsilon$ for all $x$. Let $X$ be an arbitrary set 
and $Z$ be a compact metric space. Given any two functions $p, q$ that map $X$ into $\mathcal{H}_{Z}$, let $d^{*}(p, q)=\sup _{x \in X} d(p(x), q(x))$, where $d$ is the Hausdorff metric on $\mathcal{H}_{Z}$.

Lemma 3: (i) If $p_{n} \in \mathcal{C}\left(X, \mathcal{H}_{Z}\right)$ converges to $p \in \mathcal{C}(X, Z)$ if and only if $p_{n}$ converges to $p$ uniformly; that is, $\lim _{n} d^{*}\left(p_{n}, p\right)=0$. (ii) The relative topology of $\mathcal{C}(X, Z) \subset \mathcal{C}\left(X, \mathcal{H}_{Z}\right)$ is the topology of uniform convergence.

Proof: Let $\lim p_{n}=p \in \mathcal{C}(X, Z)$. Then, $p$ is continuous and since $X$ is compact, it is uniformly continuous. For $\epsilon>0$, choose a strictly positive $\epsilon^{\prime}<\epsilon$ such that $d\left(x, x^{\prime}\right)<\epsilon^{\prime}$ implies $d\left(p(x), p\left(x^{\prime}\right)\right)<\epsilon$. Then, choose $N$ so that $d_{H}\left(G(p), G\left(p_{n}\right)\right)<\epsilon^{\prime}$ for all $n \geq N$. Hence, for $n \geq N, x \in X$ and $z \in p_{n}(x)$, we have $x^{\prime} \in X$ such that $d\left(x, x^{\prime}\right)<\epsilon^{\prime}$ and $d\left(p\left(x^{\prime}\right), z\right)<\epsilon^{\prime}$. Hence, $d(p(x), z) \leq d\left(p\left(x^{\prime}\right), z\right)+d\left(p\left(x^{\prime}\right), p(x)\right)<2 \epsilon$ as desired.

Next, we will show that $p_{n}$ converges to $p$ uniformly implies $G\left(p_{n}\right)$ converges to $G(p)$ in the Hausdorff metric. Consider any sequence $p_{n}$ converging uniformly to $p$. Choose $N$ such that $n \geq N$ implies $d\left(p_{n}(x), p(x)\right)<\epsilon$. Hence, for $n \geq N,(x, z) \in G\left(p_{n}\right)$ implies $d((x, z),(x, p(x)))<\epsilon$, proving $\varlimsup \lim G\left(p_{n}\right) \subset G(p) \subset \underline{\lim } G\left(p_{n}\right)$. This concludes the proof of (i); (ii) follows immediately from (i).

For any $\theta=\left(\theta_{0}, f_{1}, \ldots\right) \in \Theta$ let $\theta(n)=\left(\theta_{0}, f_{1}, \ldots, f_{n}\right)$ be the $n$-truncation of $\theta$. For $\theta_{n} \in \Theta_{n}$ and $n \geq 0$, let

$$
\begin{aligned}
\Theta\left(\theta_{n}\right) & =\left\{\theta \in \Theta \mid \theta(n)=\theta_{n}\right\} \\
\Theta_{n+1}\left(\theta_{n}\right) & =\left\{\left(\theta_{n}, f_{n+1}\right) \in \Theta_{n+1} \mid \theta_{n+1}=\theta_{n}\right\}
\end{aligned}
$$

Lemma 4: Let $\hat{\theta} \in \Theta \in \mathcal{I}$ with $\hat{\theta}=\left(f_{0}, f_{1}, \ldots\right)$ and $\phi(\hat{\theta})=\left(f_{0}, f\right)$. Then, for all $n \geq 1$ and $\theta_{n-1} \in \Theta_{n-1}, \bigcup_{\theta \in \Theta\left(\theta_{n-1}\right)} f(\theta)=f_{n}\left(\theta_{n-1}\right)$.

Proof: Let $P \in f_{n}\left(\theta_{n-1}\right)$. Since the sequence $\left\{\Theta_{n}\right\}$ is consistent, we can choose $\theta_{n}=$ $\left(\theta_{n-1}, g_{n}\right) \in \Theta_{n}\left(\theta_{n-1}\right)$ so that $P \in f_{n+1}\left(\theta_{n}\right)$. Repeat the argument for every $k>n$ to obtain $\theta=\left(\theta_{n-1}, g_{n}, g_{n+1}, \ldots\right) \in \Theta$ such that $\phi(\hat{\theta})(\theta)=\left(f_{0}, P\right)$. Hence, $f_{n}\left(\theta_{n-1}\right) \subset$ $\bigcup_{\theta \in \Theta\left(\theta_{n-1}\right)} f(\theta)$. That $\bigcup_{\theta \in \Theta\left(\theta_{n-1}\right)} f(\theta) \subset f_{n}\left(\theta_{n-1}\right)$ follows from the definition of $f$.

Lemma 5: Let $X, Y$ be compact metric spaces and $Z$ be an arbitrary metric space. Let $q: X \times Y \rightarrow Z$ and let the mapping $p$ from $X$ to the set of functions from $Y$ to $Z$ be defined as $p(x)(y):=q(x, y)$. Then, $q \in \mathcal{C}(X \times Y, Z)$ if and only if $p \in \mathcal{C}(X, \mathcal{C}(Y, Z))$. 
Proof: Assume $q$ is continuous. Since $X \times Y$ is compact, $q$ must be uniformly continuous. Hence, for all $\epsilon>0$ there exists $\epsilon^{\prime}>0$ such that $d\left((x, y),\left(x^{\prime}, y^{\prime}\right)\right)<\epsilon^{\prime}$ implies $d\left(q(x, y), q\left(x^{\prime}, y^{\prime}\right)\right)<\epsilon$. In particular, $d\left(x, x^{\prime}\right)<\epsilon^{\prime}$ implies $d\left(q(x, y), q\left(x^{\prime}, y\right)\right)<\epsilon$ for all $y \in Y$. Hence, $d\left(x, x^{\prime}\right)<\epsilon^{\prime}$ implies $d\left(p(x), p\left(x^{\prime}\right)\right)<\epsilon$, establishing the continuity of $p$. Next, assume that $p$ is continuous and let $\epsilon>0$. To prove that $q$ is continuous, assume $\left(x^{k}, y^{k}\right) \in X \times Y$ converges to some $(x, y) \in X \times Y$. The continuity of $p$ ensures that for some $k \in \mathbb{N}, m \geq k$ implies $d\left(p\left(x^{m}\right), p(x)\right) \leq \epsilon$. Since $p(x)$ is continuous, we can choose $k$ so that $d\left(p(x)\left(y^{m}\right), p(x)(y)\right)<\epsilon$ for all $m \geq k$ as well. Hence,

$$
d\left(p\left(x^{m}\right)\left(y^{m}\right), p(x)(y)\right) \leq d\left(p\left(x^{m}\right)\left(y^{m}\right), p(x)\left(y^{m}\right)\right)+d\left(p(x)\left(y^{m}\right), p(x)(y)\right)<2 \epsilon
$$

Lemma 6: $\quad$ Let $X$ be compact and $Z$ be an arbitrary metric space. Suppose $p \in \mathcal{C}(X, Z)$ is one-to-one. Then, $p$ is a homeomorphism from $X$ to $p(X)$.

Proof: It is enough to show that $p^{-1}: p(X) \rightarrow X$ is continuous. Take any closed $B \subset X$. Since $X$ is compact, so is $B$. Then, since the continuous image of a compact set is compact, $\left(p^{-1}\right)^{-1}(B)=p(B)$ is compact (and therefore closed). Hence, the inverse image of any closed set under $p^{-1}$ is closed and therefore $p^{-1}$ is continuous.

Lemma 7: Let $X, Y$ be a compact metric spaces. For $p \in \mathcal{C}\left(X, \mathcal{H}_{Y}\right)$, let $\bar{d}(p)=$ $\max _{x \in X} \max _{y, z \in p(x)} d(y, z)$. Then, (i) $p_{n} \in \mathcal{C}\left(X, \mathcal{H}_{Y}\right)$ converges to $p \in \mathcal{C}\left(X, \mathcal{H}_{Y}\right)$ implies $\lim \sup \bar{d}\left(p_{n}\right) \leq \bar{d}(p)$. (ii) $p, q, p^{\prime}, q^{\prime} \in \mathcal{C}\left(X, \mathcal{H}_{Y}\right)$ and $p(x) \subset p^{\prime}(x), q(x) \subset q^{\prime}(x)$ for all $x \in X$ implies $d(p, q) \leq \max \left\{d\left(p^{\prime}, q^{\prime}\right)+\bar{d}\left(p^{\prime}\right), d\left(p^{\prime}, q^{\prime}\right)+\bar{d}\left(q^{\prime}\right)\right\}$.

Proof: Since $X \times Y$ is compact, to prove (i) we can assume $p_{n} \in \mathcal{C}\left(X, \mathcal{H}_{Y}\right)$ converges to $p \in \mathcal{C}\left(X, \mathcal{H}_{Y}\right), \lim \bar{d}\left(p_{n}\right)=\alpha$ and show $\alpha \leq \bar{d}(p)$. Choose $x_{n} \in X$ and $y_{n}, z_{n} \in p_{n}\left(x_{n}\right)$ such that $d\left(y_{n}, z_{n}\right)=\bar{d}\left(p_{n}\right)$. Without loss of generality, assume $\left(x_{n}, y_{n}, z_{n}\right)$ converges to $(x, y, z)$. Hence, $\lim \bar{d}\left(p_{n}\right)=d(y, z)$. Since $p_{n}$ converges to $p, G\left(p_{n}\right)$ converges to $G(p)$. Hence, $(x, y),(x, z) \in G(p)$ by Lemma $1(\mathrm{i})$ and therefore, $\lim \bar{d}\left(p_{n}\right)=d(y, z) \leq \bar{d}(p)$.

(ii) Let $(x, z) \in G(p)$. Then,

$$
\min _{(\tilde{x}, \tilde{z}) \in G\left(q^{\prime}\right)} d((x, z),(\hat{x}, \hat{z})) \leq \min _{(\tilde{x}, \tilde{z}) \in G(q)} d((x, z),(\tilde{x}, \tilde{z}))+\bar{d}\left(q^{\prime}\right)
$$


Therefore,

$$
\min _{(\hat{x}, \hat{z}) \in G(q)} d((x, z),(\hat{x}, \hat{z})) \leq d\left(p^{\prime}, q^{\prime}\right)+\bar{d}\left(q^{\prime}\right)
$$

and a symmetric argument shows that

$$
\min _{(x, z) \in G(p)} d((x, z),(\hat{x}, \hat{z})) \leq d\left(p^{\prime}, q^{\prime}\right)+\bar{d}\left(p^{\prime}\right)
$$

Therefore, $d(p, q) \leq \max \left\{d\left(p^{\prime}, q^{\prime}\right)+\bar{d}\left(p^{\prime}\right), d\left(p^{\prime}, q^{\prime}\right)+\bar{d}\left(q^{\prime}\right)\right\}$.

\subsection{Proof of Theorem 1:}

We first show that $\phi$ is continuous. Consider any sequence $\theta^{k}=\left(f_{0}^{k}, f_{1}^{k}, \ldots\right) \in \Theta$ such that $\lim \theta^{k}=\theta=\left(f_{0}, f_{1} \ldots\right) \in \Theta$. Let $\phi(\theta)=\left(f_{0}, f\right)$ and $\phi\left(\theta^{k}\right)=\left(f_{0}^{k}, f^{k}\right)$ for all $k$. Let $\epsilon>0$. By Lemma $2, f_{n}$ converges to $f$ and therefore by Lemma $7(\mathrm{i})$, there exists $N$ such that $\bar{d}\left(f_{N}\right)<\epsilon$. Since $f_{N}^{k}$ converges to $f_{N}$, Lemma $7(\mathrm{i})$ implies that there is $k^{\prime}$ such that for $k \geq k^{\prime}, \bar{d}\left(f_{N}^{k}\right) \leq 2 \epsilon$. Also, there is $k^{\prime \prime}$ such that $d\left(f_{N}^{k}, f_{N}\right) \leq \epsilon$ for $k>k^{\prime \prime}$. Let $m=\max \left\{k^{\prime}, k^{\prime \prime}\right\}$. Lemma 7 (ii) now implies that $\left.d\left(f_{n}^{k}, f_{n}\right)\right) \leq 3 \epsilon$, for all $n \geq N$ and $k \geq m$. Therefore $d\left(f^{k}, f\right) \leq 3 \epsilon$ for all $k \geq m$. This shows that $\phi$ is continuous.

Next, we prove that $\phi$ is one-to-one. Pick any $\left(f_{0}, f_{1}, \ldots\right),\left(g_{0}, g_{1}, \ldots\right) \in \Theta$. Let $\left(f_{0}, f\right)=\phi\left(f_{0}, f_{1}, \ldots\right)$ and $\left(g_{0}, g\right)=\phi\left(g_{0}, \ldots\right)$. If $f_{0} \neq g_{0}$, then clearly $\left(f_{0}, f\right) \neq\left(g_{0}, g\right)$. Hence, assume $f_{0}=g_{0}$. Then, there exists a smallest $n \geq 1$ and $\theta_{n-1} \in \Theta_{n-1}$ such that $g_{n}\left(\theta_{n-1}\right) \neq f_{n}\left(\theta_{n-1}\right)$. By Lemma $4, \bigcup_{\theta^{\prime} \in \Theta\left(\theta_{n-1}\right)} f\left(\theta^{\prime}\right) \neq \bigcup_{\theta^{\prime} \in \Theta\left(\theta_{n-1}\right)} g\left(\theta^{\prime}\right)$ and hence $f \neq g$ as desired.

Since $\phi$ is continuous and one-to-one and $\Theta$ is compact, it follows from Lemma 6 that $\phi$ is a homeomorphism from $\Theta$ to $\phi(\Theta)$. The continuity of $\Psi$ follows from the compactness of $\Theta$ and Lemma 5 .

\subsection{Proof of Theorem 2:}

Let $M=(T, \gamma, \omega)$ be an IPM. We say that $\mathcal{D}$ is a strongly continuous partition of $T$ if the function $\sigma: T \rightarrow \mathcal{D}$ defined by $\sigma(t)=D^{t}$ is an element of $\mathcal{C}\left(T, \mathcal{H}_{T}\right)$.

Define the sequence of partitions $\mathcal{D}_{n}$ on $T$ as follows:

$$
D_{0}^{t}=\left\{t^{\prime} \in T \mid \omega\left(t^{\prime}\right)=\omega(t)\right\}
$$


and $\mathcal{D}_{0}=\left\{D_{0}^{t} \mid t \in T\right\}$. For $n \geq 1$, we define inductively

$$
D_{n}^{t}:=\left\{t^{\prime} \in D_{n-1}^{t} \mid \Gamma\left(t^{\prime}, D\right)=\Gamma(t, D) \text { for all } D \in \mathcal{D}_{n-1}\right\}
$$

and $\mathcal{D}_{n}=\left\{D_{n}^{t} \mid t \in T\right\}$. Let $\mathcal{D}=\left\{\bigcap_{n} D_{n}^{t} \mid t \in T\right\}$ and note that $\mathcal{D}$ is a partition of $T$.

Step 1: (i) Each $\mathcal{D}_{n}$ is strongly continuous. (ii) $M$ is valid if and only if $\mathcal{D}=\{\{t\} \mid t \in T\}$.

Proof: (i) The proof is by induction. Assume that $t_{k}$ converges to $t, \hat{t}_{k} \in D_{0}^{t_{k}}$ and $\hat{t}_{k}$ converges to $\hat{t}$. Then, $\omega(\hat{t})=\lim \omega\left(\hat{t}_{k}\right)=\lim \omega\left(t_{k}\right)=\omega(t)$. Hence, $\hat{t} \in D_{0}^{t}$. For $t_{k}=t$ for all $k$, the preceding argument shows that $D_{0}^{t}$ is closed and hence compact. Then, the general case proves the strong continuity of $\mathcal{D}_{0}$.

Assume that $\mathcal{D}_{n}$ satisfies strong continuity. Hence, every $D \in \mathcal{D}_{n}$ is compact. Next, assume that $t_{k}$ converges to $t, \hat{t}_{k} \in D_{n+1}^{t_{k}}$ and $\hat{t}_{k}$ converges to $\hat{t}$. Hence, $\hat{t}_{k} \in D_{n}^{t_{k}}$ and by the strong continuity of $\mathcal{D}_{n}$, we have $\hat{t} \in D_{n}^{t}$. Pick any $D \in \mathcal{D}_{n}$ and $P \in \Gamma(\hat{t}, D)$. By, Lemma 1(i) and (ii), we have a subsequence $k_{j}$ and $P_{k_{j}} \in \Gamma\left(\hat{t}_{k_{j}}, D\right)=\Gamma\left(t_{k_{j}}, D\right)$ such that $\lim P_{k_{j}}=P$. Then, again by Lemma 1(i) and (ii), we have $P \in \Gamma(t, D)$, proving that $\Gamma(\hat{t}, D) \subset \Gamma(t, D)$. A symmetric argument ensures that $\Gamma(\hat{t}, D)=\Gamma(t, D)$, establishing that $\hat{t} \in D_{n+1}^{t}$. For $t_{k}=t$ for all $k$, the preceding argument shows that $D_{n+1}^{t}$ is closed and hence compact. Then, the general case proves the strong continuity of $\mathcal{D}_{n+1}$. This concludes the proof of part (i).

If $\mathcal{D} \neq\{\{t\} \mid t \in T\}$, then $M$ is not valid. Suppose $M$ is not valid and hence there exists a partition $\mathcal{D}_{*}$ other than the finest partition that satisfies the condition stated in the definition of validity. Then, we claim that $\mathcal{D}_{*}$ is a refinement of $\mathcal{D}$; that is, $D_{*}^{t} \in \mathcal{D}_{*}$ and $D^{t} \in \mathcal{D}$ implies $D_{*}^{t} \subset D^{t}$. To see this note that since $\mathcal{D}_{*}$ satisfies the condition stated the definition of validity, it is a refinement of $\mathcal{D}_{0}$. Moreover, if $\mathcal{D}_{*}$ is a refinement of $\mathcal{D}_{n}$ then $\mathcal{D}_{*}$ is a refinement of $\mathcal{D}_{n+1}$. The last assertion follows from the fact that for $t^{\prime} \in D_{*}^{t} \in \mathcal{D}_{*}$,

$$
\Gamma\left(t, D_{n}\right)=\bigcup_{D \in \mathcal{D}_{*}, D \subset D_{n}} \Gamma(t, D)=\bigcup_{D \in \mathcal{D}_{*}, D \subset D_{n}} \Gamma\left(t^{\prime}, D\right)=\Gamma\left(t^{\prime}, D_{n}\right)
$$

Hence, $\mathcal{D}_{*}$ is a refinement of $\mathcal{D}_{n}$ for all $n \geq 0$. Therefore, $D_{*}^{t} \in \mathcal{D}_{*}$ implies

$$
D_{*}^{t} \subset\left\{\bigcap_{n} D_{n}^{t} \mid t \in T\right\}=D^{t} \in \mathcal{D}
$$


Since, $\mathcal{D}_{*}$ is not the finest partition, neither is $\mathcal{D}$. This concludes the proof of step 1 .

Let $(T, \Gamma, \omega)$ be an IPM. Define, $\Theta_{0}:=\Omega=\omega(T), f_{0}^{t}:=\omega(t)$ and $\iota_{0}(t):=f_{0}^{t}$ for all $t \in T$. Then, inductive define $f_{n}^{t}: \Theta_{n-1} \rightarrow \mathcal{H}, \Theta_{n}, \iota_{n}: T \rightarrow \Theta_{n}$ as follows:

$$
\begin{aligned}
f_{n}^{t}\left(\theta_{n-1}\right) & =\Gamma\left(t, \iota_{n-1}^{-1}\left(\theta_{n-1}\right)\right) \\
\iota_{n}(t) & =\left(\iota_{n-1}(t), f_{n}^{t}\right) \\
\Theta_{n} & =\iota_{n}(T)
\end{aligned}
$$

Let

$$
\begin{aligned}
\Theta & =\left\{\left(f_{0}, f_{1}, \ldots\right) \mid\left(f_{0}, f_{1}, \ldots, f_{n}\right) \in \Theta_{n} \text { for all } n \geq 0\right\} \\
\iota(t) & =\left(f_{0}, f_{1}, \ldots\right) \text { such that }\left(f_{0}, f_{1}, \ldots, f_{n}\right)=\iota_{n}(t) \text { for all } n .
\end{aligned}
$$

Henceforth, for any $t \in T$ such that $\iota(t)=\left(f_{0}, f_{1}, \ldots\right)$, let $f_{n}^{t}$ denote the corresponding $f_{n}$. We define the functions $g_{n}^{t}: T \rightarrow \mathcal{H}$ as follows:

$$
g_{n}^{t}(s)=\Gamma\left(t, D_{n-1}^{s}\right)
$$

Step 2: $\quad$ For all $n \geq 0$, the sets $\Theta_{n}$ is non-empty and compact and the function $\iota_{n}$ is onto and continuous; for $n>0, \Theta_{n} \subset \mathcal{C}\left(\Theta_{n-1}, \mathcal{H}\right)$.

Proof: Clearly, $\Theta_{0}$ is nonempty and compact and the function $\iota_{0}$ is onto and continuous. We will prove inductively that for all $n>0, \Theta_{n}$ is a nonempty, compact subset of $\mathcal{C}\left(\Theta_{n-1}, \mathcal{H}\right)$ and $\iota_{n}$ is continuous and onto. By Lemma 1(iv), the continuity of $\Gamma$ and $\iota_{n-1}$ ensures that $\Theta_{n} \subset \mathcal{C}\left(\Theta_{n-1}, \mathcal{H}\right)$. Since $T$ is nonempty, so is $\Theta_{n}$. The function $\iota_{n}$ is onto by definition. Since $\iota_{n-1}$ is continuous, the continuity of $\Gamma$ and Lemma 1(iii), (iv) imply that the function $\left(t, \theta_{n-1}\right) \rightarrow f_{n}^{t}\left(\theta_{n-1}\right)$ is also continuous. Then, by Lemma 5 , the function $t \rightarrow f_{n}^{t}$ is also continuous and hence $\iota_{n}$ is continuous. Since $\Theta_{n-1}$ is nonempty and compact, so is $\Theta_{n}$.

Step 3: (i) The function $\iota$ is onto. (ii) $\iota_{n}(t)=\iota_{n}(s)$ if and only if $D_{n}^{t}=D_{n}^{s}$. (iii) $f_{n}^{t}\left(\iota_{n-1}(s)\right)=g_{n}^{t}(s)$.

Proof: First, we show that $\iota: T \rightarrow \Theta$ is onto. Pick $\left(f_{0}, f_{1}, \ldots\right)$ such that $\left(f_{0}, f_{1}, \ldots, f_{n}\right) \in$ $\Theta_{n}$ for all $n$. Then, for all $n$, there exists $t_{n} \in T$ such that $\iota_{n}\left(t_{n}\right)=\left(f_{0}, f_{1}, \ldots, f_{n}\right)$. 
Take $t_{n_{j}}$, a convergent subsequence of $t_{n}$ converging to some $t \in T$. Then, for all $j$, $\iota_{j}\left(t_{n_{j}}\right)=\left(f_{0}, f_{1}, \ldots, f_{j}\right)$. Hence, the continuity of $\iota_{j}$ ensures that $\iota_{j}(t)=\left(f_{0}, f_{1}, \ldots, f_{j}\right)$ for all $j$, establishing that $\iota(T)=\Theta$.

Next, we prove that $\iota_{n}(t)=\iota_{n}(s)$ if and only if $D_{n}^{t}=D_{n}^{s}$. To see this, note that for $n=0$, the assertion is true by definition. Suppose, it is true for $n$. Then, if $s \in D_{n+1}^{t}$, we have $s \in D_{n}^{t}$ and $\Gamma(t, D)=\Gamma(s, D)$ for all $D \in \mathcal{D}_{n}$. Hence, $f_{n+1}^{t}=f_{n+1}^{s}$ and therefore, by the inductive hypothesis, $\iota_{n+1}(t)=\iota_{n+1}(s)$. Conversely, if $\iota_{n+1}(t)=\iota_{n+1}(s)$, then $f_{n+1}^{t}=f_{n+1}^{s}$ and $\iota_{n}(t)=\iota_{n}(s)$. Then, by the inductive hypothesis, $s \in D_{n}^{t} \in \mathcal{D}_{n}$ and also, $\Gamma(t, D)=\Gamma(s, D)$ for all $D \in \mathcal{D}_{n}$; that is, $s \in D_{n+1}^{t} \in \mathcal{D}_{n+1}$ as desired.

Part (iii) follows from part (ii) and the definitions of $g_{n}^{t}, f_{n}^{t}$.

Step 4: $M$ is isomorphic to some $\Theta \in \mathcal{I}$ if and only if $\mathcal{D}=\{\{t\} \mid t \in T\}$.

Assume $\mathcal{D}=\{\{t\} \mid t \in T\}$. We will show that the function $\iota$ defined above is the desired isomorphism. Clearly, $\left\{\Theta_{0}, \Theta_{1}, \ldots\right\}$ is a system of best response hierarchies. Step 3 (ii) implies that $\Theta_{n-1}\left(\theta_{n-2}\right)=\iota_{n-1}\left(D_{n-2}^{s}\right)$ for $s$ such that $\iota_{n-2}(s)=\theta_{n-2}$. Therefore,

$$
f_{n-1}^{t}\left(\theta_{n-2}\right)=\Gamma\left(t, D_{n-2}^{s}\right)=\bigcup_{s^{\prime} \in D_{n-2}^{s}} \Gamma\left(t, D_{n-1}^{s^{\prime}}\right)=\bigcup_{\theta_{n-1}^{\prime} \in \Theta_{n-1}\left(\theta_{n-2}\right)} f_{n}^{t}\left(\theta_{n-1}^{\prime}\right)
$$

proving that $\left\{\Theta_{n}\right\}$ satisfies the consistency condition (C1); (C2) follows immediately from the symmetry built-in to the definition of an IPM.

Let $f^{t}: \Theta \rightarrow \mathcal{H}$ be defined by

$$
f^{t}(\theta)=\bigcap_{n \geq 1} f_{n}^{t}(\theta)
$$

Since, $\iota_{n}(t)=\iota_{n}(s)$ if and only if $D_{n}^{t}=D_{n}^{s}$ (Step 3(ii)), we conclude that $\iota$ is one-to-one. For $\hat{\theta}=\left(\hat{f}_{0}, \hat{f}_{1}, \ldots\right)$ we let $\hat{\theta}(n)$ be defined as $\left(\hat{f}_{0}, \ldots, \hat{f}_{n}\right)$. By Step $3, f_{n}^{t}(\hat{\theta}(n-1))=$ $g_{n}^{t}(s)=\Gamma\left(t, D_{n}^{s}\right)$ for $\hat{\theta}=\iota(s)$. Then, since the IPM $(T, \Gamma, \omega)$ is valid, Step 1(ii) implies that $f^{t}(\hat{\theta})=\bigcap_{n} \Gamma\left(t, D_{n}^{s}\right)=\Gamma(t, s)$ and therefore $f^{t}(\hat{\theta})$ is a singleton.

To prove that $\iota$ is a homeomorphism, we prove that $\iota$ is continuous and appeal to Lemma 6. Consider the mapping $\hat{\theta} \rightarrow \iota_{n}^{-1}(\hat{\theta}(n-1))$. Clearly, $\hat{\theta} \rightarrow(\hat{\theta})$ and by Step 2, $\iota_{n}^{-1}$ is continuous. Then, it is easy to verify that $\hat{\theta} \rightarrow \iota_{n}^{-1}(\hat{\theta}(n-1))$ is also continuous which by 
Lemma 1 (iii) implies $(t, \hat{\theta}) \rightarrow f_{n}^{t}\left(\hat{\theta}_{n-1}\right)$ is continuous. Let this mapping be $I_{n}: T \times \Theta \rightarrow \mathcal{H}$. Let $I$ be the mapping that takes $(t, \hat{\theta})$ to $f^{t}(\hat{\theta})=\bigcap_{n} I_{n}(\theta)$. By Lemma $2(\mathrm{i}), I$ is continuous and therefore, by Lemma $5, \iota$, the mapping that takes $t$ to $I(t, \cdot)=\iota(t)$ is also continuous and therefore $\iota$ is a homeomorphism.

Next we will show that if $M$ is isomorphic to some $\Theta$, then $\mathcal{D}=\{\{t\} \mid t \in T\}$. Assume that $\hat{\iota}$ is the desired isomorphism between $M$ and $\Theta$. Define the sequence of partitions $\hat{D}_{n}$ as above with each $f=\hat{\imath}(t) \in \Theta$ replacing the corresponding $t$. A simple inductive argument establishes that $\hat{D}_{n}^{\hat{\iota}(t)}=D_{n}^{t}$ for all $t, n$. Let $T^{\prime}=\Theta$ and argue as in the proof of step 3 above to conclude that $\left(\hat{f}_{0}, \ldots, \hat{f}_{n}\right) \in D_{n}^{\theta}$ if and only if $\hat{f}_{n}=f_{n}$ for $\theta_{n}=\left(f_{0}, f_{1}, \ldots\right)$. Now, suppose $s \neq t$ and hence $\left(\hat{f}_{0}, \hat{f}_{1}, \ldots\right)=\hat{\iota}(s) \neq \hat{\iota}(t)=\left(f_{0}, f_{1}, \ldots\right)$ and hence $\hat{\iota}(s) \notin \hat{D}_{n}^{\hat{\iota}(t)}$ for some $n$ proving that $\{\theta\}=\bigcap_{n} D_{n}^{\theta}$ for all $\theta$; that is, $\{t\}=\bigcap_{n} D_{n}^{t}$ for all $t$.

Suppose $M$ is a valid IPM. Then, Step 1(ii) implies that $\mathcal{D}=\{\{t\} \mid t \in T\}$ and hence Step 4 implies that $M$ is isomorphic to some component of the canonical type space. Conversely, suppose that the IPM $M$ is isomorphic to a component of the canonical type space. Then, Steps 4 and 1 imply that $M$ is valid.

\section{Appendix C: Proof of Theorem 3}

Fix the IPM $(T, \gamma)$ with $T$ finite and of cardinality $k$. Define the sequence of partitions $\mathcal{D}_{n}$ on $T$ as follows: $\mathcal{D}_{0}=\{T\}$. For $n \geq 1$, we define inductively

$$
D_{n}^{t}:=\left\{t^{\prime} \in D_{n-1}^{t} \mid \Gamma\left(t^{\prime}, D\right)=\Gamma(t, D) \text { for all } D \in \mathcal{D}_{n-1}\right\}
$$

and $\mathcal{D}_{n}=\left\{D_{n}^{t} \mid t \in T\right\}$. It is easy to show that $\mathcal{D}_{n}$ is a refinement of $\mathcal{D}_{n-1}$. For any $\mathcal{D}$, let $\ell(\mathcal{D})=\#(T)-\#(\mathcal{D})$, where $\#(X)$ is the cardinality of the set $X$.

Lemma 8: $\quad \ell\left(\mathcal{D}_{k-1}\right)=0$ if and only if $(T, \gamma)$ is valid.

Proof: First, we show that if $(T, \gamma)$ is valid, then $\ell\left(\mathcal{D}_{k-1}\right)=0$. Clearly, $\ell\left(\mathcal{D}_{0}\right)=k-1$. To conclude this part of the proof, we will claim that $\ell\left(\mathcal{D}_{n}\right)<\ell\left(\mathcal{D}_{n-1}\right)$ whenever $\ell\left(\mathcal{D}_{n-1}\right)>0$. Since $(T, \gamma)$ is valid, it follows that $\mathcal{D}_{n}$ is a refinement of $\mathcal{D}_{n-1}$ and $\mathcal{D}_{n} \neq \mathcal{D}_{n-1}$. Therefore, there are $D_{1}, D_{2} \in \mathcal{D}_{n}$ such that $D_{1} \cup D_{2} \subset D_{n-1}$. Hence, $\ell\left(\mathcal{D}_{n}\right) \leq \ell\left(\mathcal{D}_{n-1}\right)-1$ as desired. 
For the "only if" part, suppose $\ell\left(\mathcal{D}_{k-1}\right)>0$. Then, $\mathcal{D}_{k-1}$ is not the finest partition and we must have $\mathcal{D}_{n}=\mathcal{D}_{n-1}$ for some $n \leq k-1$. Hence, $\mathcal{D}_{n}=\mathcal{D}_{k-1}$ and $\mathcal{D}_{n}$ is the partition that establishes the failure of validity.

\section{Proof of Theorem 3:}

To prove the "only if" part, assume that $(T, \gamma)$ is not valid and let $\mathcal{D}$ be a partition such that $\ell(\mathcal{D})>0$ and $\Gamma(t, D)=\Gamma\left(t^{\prime}, D\right)$ for all $t, t^{\prime} \in D^{t} \in \mathcal{D}$ and all $D \in \mathcal{D}$. Hence, there exists $D \in \mathcal{D}$ such that $t \neq t^{\prime}$ and $t, t^{\prime} \in D$. Without loss of generality, let $T=\{1, \ldots, k\}$ and $t=1$ and $t^{\prime}=2$.

We will construct a 1-complete sample. To describe the sample, we first construct the following complete information game tree: the initial node is 1 and has $k$ successors, $(1, t)$ for $t \in T$. Each of the nodes $(1, t)$ has $k-1$ successors $(1, t, s)$ for $s \in T \backslash\{1\}$; each of these nodes again has $k-1$ successors $\left(1, t, s, s^{\prime}\right)$ such that $s^{\prime} \in T \backslash\{t\}$ and so on for $k-1$ rounds so that the set of terminal nodes is

$$
\left\{x \in\{1\} \times T^{k-1} \mid x=\left(s_{0}, s_{1}, \ldots, s_{m}\right) \text { implies } s_{i-1} \neq s_{i+1} \text { for all } i\right\}
$$

We interpret each node as a distinct player and hence identify $N$ with the set of nodes. We identify each edge that connects a node with its predecessor with an interaction. Hence, player 1 interacts with players $(1, t)$ for all $t \in T$, player $(1, t)$ interacts with player 1 and players $(1, t, s)$ for all $s \in T \backslash\{1\}$ and so on. Clearly, this sample is 1-complete.

Let $\tau$ be the following prediction for this sample: $\tau(1)=2, \tau(1, t)=t^{\prime}$ for any $t^{\prime} \in D^{t}$ such that $\Gamma\left(2, t^{\prime}\right)=\Gamma(1, t)$ for all $t \in T ; \tau(1, t, s)=t^{\prime}$ for any $t^{\prime} \in D^{t}$ such that $\Gamma\left(\tau(1, t), t^{\prime}\right)=\Gamma(t, s) ; \tau\left(1, t, s, s^{\prime}\right)=t^{\prime}$ for any $t^{\prime} \in D^{s^{\prime}}$ such that $\Gamma\left(\tau(1, t, s), t^{\prime}\right)=\Gamma\left(s, s^{\prime}\right)$ and so on. Clearly, this is possible since $\Gamma(s, D)=\Gamma(t, D)$ for all $s \in D^{t} \in \mathcal{D}$. By construction $\tau$ is consistent but $\tau(1) \neq t_{1}$. This proves the "only if" part of the theorem.

Next, we prove the "if" part: again without loss of generality, assume $i=1$ and hence $\Sigma$ is a 1-complete sample. Since $\Sigma$ is 1-complete it must contain a tree of interactions such as the one describe above possibly with the same player occupying multiple nodes. For any consistent match, we must have $\tau(x) \in D_{1}^{x}$ for all $x \in\{1\} \times T^{k-1}$. By induction, we must have $\tau(x) \in D_{j}^{x}$ for all $x \in\{1\} \times T^{k-j}$ and hence $1 \in D_{k-1}^{1}=\{1\}$ proving that types are observable in $(T, \gamma)$. 


\section{Appendix D: Proof of Theorem 4}

Section 4.3 sketches the definition of communicability. Here we provide a formal definition. Let $(T, \gamma)$ be an IPM with a single characteristic and a finite set of types. In the equivalent epistemic model, each player knows his own type and knows nothing about his opponent's type; that is, any type in $T$ possible. Formally, the epistemic model $E=\left\{S, \mathcal{T}_{1}, \mathcal{T}_{2}\right\}$ is equivalent to the $\operatorname{IPM}(T, \gamma)$ if $S=T \times T, \mathcal{T}_{1}=\{\{t\} \times T \mid t \in T\}$ and $\mathcal{T}_{2}=\{T \times\{t\} \mid t \in T\}$.

A collection of subsets $\mathcal{L}$ of a set $S$ is an algebra (or equivalently, a language) if it contains $S$ and is closed under unions and complements. For any two algebras $\mathcal{K}, \mathcal{L}$, let $\mathcal{K} \vee \mathcal{L}$ denote the smallest (in terms of set inclusion) algebra that contains both and let $\mathcal{K} \wedge \mathcal{L}$ be the largest that is contained in both.

Definition: The language of preferences is $\mathcal{L}_{p}=\left\{\Gamma^{-1}(V) \mid V \in \mathcal{H}\right\}$.

Player 1, with knowledge $\mathcal{T}_{1}$, can use the word $B \in \mathcal{L}_{p}$ to make a statement about whether or not he knows $B$. Let

$$
\mathcal{T}_{1} * B:=\bigcup_{A \in \mathcal{T}_{1}, A \subset B} A
$$

Hence, player 1 can communicate $\mathcal{T}_{1} * B$ for all $B \in \mathcal{L}_{p}$ to player 2 . He can also communicate what he does not know. Then, using standard logical operations, player 2 can attain the level of knowledge described by the smallest partition consistent with the statements:

$$
\mathcal{T}_{1} * \mathcal{L}_{p}=\bigvee\left\{\mathcal{A} \mid \mathcal{T}_{1} * A \subset \mathcal{A} \text { forall } A \in \mathcal{L}\right\}
$$

where $\bigvee\left\{\mathcal{K}_{1}, \ldots, \mathcal{K}_{m}\right\}:=\mathcal{K}_{1} \vee \cdots \vee \mathcal{K}_{m}$. Finally, player 2 can combine what she learned with what she already knows, $\mathcal{T}_{2}$, to attain knowledge $\hat{\mathcal{T}}_{2}$ :

$$
\hat{\mathcal{T}}_{2}=\mathcal{T}_{2} \vee\left(\mathcal{T}_{1} * \mathcal{L}_{p}\right)
$$

Let $\Lambda$ be the collection of all algebras. This set is a lattice under set inclusion and the set $\Lambda_{o}=\left\{\mathcal{T} \in \Lambda \mid \mathcal{T}_{1} \subset \mathcal{T}\right\}$ is a sublattice of $\Lambda$. For any $A \subset S$, let $A^{\top}=\left\{\left(t^{\prime}, t\right) \mid\left(t, t^{\prime}\right) \in A\right\}$ 
and for any $\mathcal{T} \in \Lambda$, let $\mathcal{T}^{\top}=\left\{A^{\top} \mid A \in \mathcal{T}\right\}$. Note that $\mathcal{T}_{2}=\mathcal{T}_{1}^{\top}$. Define the function $F: \Lambda_{o} \rightarrow \Lambda_{o}$ as follows:

$$
F(\mathcal{T})=\mathcal{T} \vee\left(\mathcal{T}^{\top} * \mathcal{L}_{p}\right)
$$

Hence, $F\left(\mathcal{T}_{1}\right)$ describes player 1's knowledge after one round of communication in which the two players simultaneously convey everything they can with language $\mathcal{L}_{p}$. The symmetry of $\Gamma$ and hence $\mathcal{L}_{p}$ ensures that $\left(F\left(\mathcal{T}_{1}\right)\right)^{\top}$ will describe player 2 's knowledge after this one round of communication.

Let $\mathcal{T}_{* *}$ be the finest partition of $S$. Clearly, $\mathcal{T}_{* *}$ is a the largest fixed-point of $F$. Note that $F$ is an increasing function; that is, $\mathcal{T} \subset \hat{\mathcal{T}}$ implies $F(\mathcal{T}) \subset F(\hat{\mathcal{T}})$. Therefore, $F$ has a smallest fixed-point; that is, a fixed-point $\mathcal{T}_{*}$ such that $\mathcal{T}_{*} \subset \mathcal{T}$ whenever $\mathcal{T}=F(\mathcal{T})$. It is easy to verify that the smallest fixed-point of $F$ can be computed as follows: let $\mathcal{T}^{1}=\mathcal{T}_{1}$ and $\mathcal{T}^{n}=F\left(\mathcal{T}^{n-1}\right)$ for all $n>1$. The finiteness of $\Lambda$ and the increasingness of $F$ ensure that $\mathcal{T}^{n}=\mathcal{T}^{n-1}$ for some $n$ and that $\mathcal{T}^{n}$ is the smallest fixed-point of $F$.

We claim that if $\mathcal{T}_{*} \neq \mathcal{T}_{* *}$, then players cannot learn each other's type by communicating in the language of preferences irrespective of the protocol; that is, even if players do not convey everything they can in each round. To see why this is the case, note that

since $\mathcal{T}_{1} \subset \mathcal{T}_{*}$ and $\mathcal{T}_{2}^{\top} \subset \mathcal{T}_{*}^{\top}$, after the initial round of communication, player 1 can only reach a level of knowledge $\mathcal{T} \subset F\left(\mathcal{T}_{*}\right)=\mathcal{T}_{*}$ and player 2 can reach a level of knowledge $\hat{\mathcal{T}} \subset\left(F\left(\mathcal{T}_{*}\right)\right)^{\top}=\mathcal{T}_{*}^{\top}$ which will ensure that after the next round, player 1's knowledge will be no better than $\mathcal{T}_{*}$ and player 2 's knowledge will again be no better than $\mathcal{T}_{*}^{\top}$ and so on. Conversely, if $\mathcal{T}_{* *}$ is the only fixed-point of $F$, then communication can continue until players learn each others' type. This observation motivates the following definition:

Definition: $\quad \operatorname{An} \operatorname{IPM}\left(S, \mathcal{T}_{1}, \mathcal{T}_{2}, \Gamma\right)$ in epistemic form is communicable in language preferences if $\mathcal{T}_{*}=\mathcal{T}_{* *}$.

\section{Proof of Theorem 4:}

For $\mathcal{T} \in \Lambda_{o}$, let $\mathcal{D}(\mathcal{T})=\{D \mid(t, D) \in \mathcal{T}$ for some $t \in T\}$. Conversely, for any partition $\mathcal{D}$ of $T$, there exists some $\mathcal{T} \in \Lambda_{o}$ such that $\mathcal{D}=\mathcal{D}(\mathcal{T})$. Note that $\mathcal{T} \neq \mathcal{T}_{* *}$ if and only if $\mathcal{D}(\mathcal{T})$ is not the finest partition. 
Suppose $(T, \gamma)$ is not valid and let $\mathcal{D} \neq \mathcal{D}\left(\mathcal{T}_{* *}\right)$ be such that $\Gamma\left(t^{\prime}, D\right)=\gamma(t, D)$ for all $t \in T$ and $t^{\prime} \in D^{t}$. Let $\mathcal{T}$ be the algebra such that $\mathcal{D}=\mathcal{D}(\mathcal{T})$; that is, $\mathcal{T}=\{(t, D) \mid t \in$ $T, D \in \mathcal{D}\}$. Hence, $\mathcal{T} \neq \mathcal{T}_{* *}$. We claim that $\mathcal{T}$ is a fixed point of $F$. To see this, note that the condition $\left[t^{\prime} \in D^{t}\right.$ implies $\Gamma(t, D)=\Gamma\left(t^{\prime}, D\right)$ for all $\left.D \in \mathcal{D}\right]$ means that $t^{\prime}$ can make exactly the same preference statements as $t$. Hence, $\mathcal{T} * B=\left\{D \times D^{\prime} \mid D \times D^{\prime} \subset B\right\}$ for all $B \subset S$ and therefore,

$$
F(\mathcal{T})=\mathcal{T} \vee\left(\mathcal{T}^{\top} * \mathcal{L}_{p}\right)=\mathcal{T} \vee\left\{D \times D^{\prime} \mid D, D^{\prime} \in \mathcal{D}\right\}=\mathcal{T}
$$

proving that $\mathcal{T}$ is a fixed point of $F$. Hence, $\mathcal{T}_{*} \neq \mathcal{T}_{* *}$ as desired.

To prove the converse, suppose $\mathcal{T}_{*} \neq \mathcal{T}_{* *}$. Then, $\mathcal{D}\left(\mathcal{T}_{*}\right)$ is not the finest partition of $T$ and, since $\mathcal{T}_{*}$ is a fixed point of $F$, we must have

$$
F\left(\mathcal{T}_{*}\right)=\mathcal{T}_{*} \vee\left(\mathcal{T}_{*}^{\top} * \mathcal{L}_{p}\right)=\mathcal{T}_{*} \vee\left\{D \times D^{\prime} \mid D, D^{\prime} \in \mathcal{D}\left(\mathcal{T}_{*}\right)\right\}=\mathcal{T}_{*}
$$

This can only happen if $\Gamma\left(t^{\prime}, D\right)=\Gamma(t, D)$ for all $t \in T, t^{\prime} \in D^{t}$ and $D \in \mathcal{D}\left(\mathcal{T}_{*}\right)$; that is, if $(T, \gamma)$ is not valid. To see why, assume $\Gamma\left(t^{\prime}, D\right) \neq \Gamma(t, D)$ for some $t \in T, t^{\prime} \in D^{t}$ and $D \in \mathcal{D}\left(\mathcal{T}_{*}\right)$. Then, there are two possibilities: (i) $\Gamma\left(t^{\prime}, D\right)$ is not a subset of $\Gamma(t, D)$ or (ii) $\Gamma(t, D)$ is not a subset of $\Gamma\left(t^{\prime}, D\right)$. If (i) holds then, in any epistemic state $(t, \hat{t})$ for $\hat{t} \in D$,

player 1 could make the statement "I know $\Gamma(t, D)$ " to distinguish himself from epistemic type $\left(t^{\prime}, D\right)$ and improve player 2's knowledge, contradicting the fact that $\mathcal{T}^{*}$ is a fixed point of $F$; if (ii) holds, then player 1 could make the statement "I don't know $\Gamma\left(t^{\prime}, D\right)$ " to achieve the same effect.

\section{Appendix E: Proof of Theorems 5 and 6}

For any ordered IPM $(T, \delta)$, let $\Delta\left(t, t^{\prime}\right)=\left(\delta\left(t, t^{\prime}\right), \delta\left(t^{\prime}, t\right)\right)$. Then, the definition of validity for an ordered IPM replaces $\Gamma$ with $\Delta$.

Lemma 9: Let $M=(T, \delta)$ be a simple IPM and suppose $\delta$ is nondecreasing in both arguments. Then, $M$ is a reciprocity model if and only if $\delta(x, \cdot)=\delta(z, \cdot)$ and $\delta(\cdot, x)=\delta(\cdot, z)$ implies $x=y$.

Proof: Let $M=(T, \delta)$ be a simple IPM and assume $\delta$ is nondecreasing in both arguments but $\delta(x, \cdot)=\delta(z, \cdot)$ and $\delta(\cdot, x)=\delta(\cdot, z)$ for some $x \neq y$. Then, define the partition $\mathcal{D}$ as 
follows: for $y \notin\{x, z\}, D^{y}=\{y\}$ and $D^{x}=D^{z}=\{x, z\}$. It follows that $\delta(x, \cdot)=\delta(z, \cdot)$ and therefore $\Delta(w, D)=\Delta\left(w^{\prime}, D\right)$ for all $w \in T, w^{\prime} \in D^{w}$, and $D \in \mathcal{D}$. Hence, $M$ is not valid.

Next, suppose that $M$ is not valid. Then, there exists a partition $\mathcal{D}$ of $T$ such that (i) there is $D \in \mathcal{D}$ and $x, z \in D$ such that $x \neq z$, (ii) $\Delta(w, D)=\Delta\left(w^{\prime}, D\right)$ for all $w \in T$, $w^{\prime} \in D^{w}$, and $D \in \mathcal{D}$. Let $\bar{D}$ denote the closure of $D$. The continuity of $\Delta$ ensures that

$$
\Delta\left(w, \bar{D}_{2}\right)=\Delta\left(w^{\prime}, \bar{D}_{2}\right)
$$

for all $w, w^{\prime} \in \bar{D}_{1}$ and $D_{1}, D_{2} \in \mathcal{D}$. To see this, take $w, w^{\prime} \in \bar{D}_{1}$ and $y \in \bar{D}_{2}$. By definition, there exists a sequence $\left(w_{n}, w_{n}^{\prime}, y_{n}\right) \in D_{1} \times D_{1} \times D_{2}$ converging to $\left(w, w^{\prime}, y\right)$. Moreover, there exists $y_{n}^{\prime} \in D_{2}$ such that $\Delta\left(w_{n}, y_{n}\right)=\Delta\left(w_{n}^{\prime}, y_{n}^{\prime}\right)$ for all $n$. Since $\bar{D}_{2}$ is compact, $y_{n}^{\prime}$ has a convergent subsequence that converges to some $y^{\prime} \in \bar{D}_{2}$. Assume, without loss of generality, that this subsequence is $y_{n}^{\prime}$ itself. Then, the continuity of $\Delta$ ensures $\Delta(w, y)=\Delta\left(w^{\prime}, y^{\prime}\right)$ and proves $(A 4)$.

The weak monotonicity of $\delta$ in both arguments together with (A4) imply

$$
\begin{aligned}
\Delta\left(\max \bar{D}_{1}, \max \bar{D}_{2}\right) & =\Delta\left(\min \bar{D}_{1}, \max \bar{D}_{2}\right) \\
\Delta\left(\min \bar{D}_{1}, \min \bar{D}_{2}\right) & =\Delta\left(\max \bar{D}_{1}, \min \bar{D}_{2}\right)
\end{aligned}
$$

Appealing to the symmetry $\Delta$ and reversing the roles of $\bar{D}_{1}$ and $\bar{D}_{2}$ in the argument leading to the second equation above yields $\Delta\left(\min \bar{D}_{1}, \min \bar{D}_{2}\right)=\Delta\left(\min \bar{D}_{1}, \max \bar{D}_{2}\right)$. Hence,

$$
\Delta\left(\max \bar{D}_{1}, \max \bar{D}_{2}\right)=\Delta\left(\min \bar{D}_{1}, \min \bar{D}_{2}\right)
$$

Then, monotonicity of $\Delta$ ensures $\delta(w, y)=\delta\left(w^{\prime}, y\right)$ for all $y \in T$ whenever $w, w^{\prime} \in \bar{D}$, in particular, for $w=x$ and $w^{\prime}=z$. One more appeal to the symmetry of $\Delta$ yields $\delta(y, x)=\delta(y, z)$ for all $y \in T$ as desired.

\subsection{Proof of Theorem 5}

Lemma 9 establishes that any simple increasing IPM is a reciprocity model. Next, suppose $M=(T, \delta)$ is a reciprocity model. By definition, $\succeq$, the nicer than relation is transitive and since $M$ is reciprocity model, it is also complete. The continuity of 
$\gamma$ yields the continuity of $\succeq$. Since $T$ is a compact metric space, it is separable and hence there exists a continuous real-valued function $x: T \rightarrow \mathbb{R}$ that represents $\succeq$ (See for example, the proposition in Jaffray (1975)). Let $K:=x(T)=\{x(t) \mid t \in T\}$. Let $D^{t}=\left\{t^{\prime} \in T \mid x\left(t^{\prime}\right)=x(t)\right\}$ and $\mathcal{D}=\left\{D^{t} \mid t \in T\right\}$. Clearly, $\mathcal{D}$ is a partition of $T$ such that $\delta\left(t^{\prime}, \cdot\right)=\delta(t, \cdot)$ for all $t^{\prime} \in D^{t}$. Since every type reciprocates, $\delta\left(\cdot, t^{\prime}\right)=\delta(\cdot, t)$ for all $t^{\prime} \in D^{t}$ and therefore $\Delta(t, D)=\Delta\left(t^{\prime}, D\right)$ for all $t \in T, t^{\prime} \in D^{t}$ and $D \in \mathcal{D}$. Since $M$ is valid, each $D^{t}$ is a singleton and therefore $x$ is one-to-one. Then, the compactness of $T$ ensures that $K$ is compact and that $x$ is a homeomorphism. Define, $\delta^{\prime}(w, y)=\delta\left(x^{-1}(w), x^{-1}(y)\right)$ for all $w, y \in K$. Since $x$ and $\delta$ are continuous, so is $\delta^{\prime}$. It follows that $\left(K, \delta^{\prime}\right)$ is isomorphic to $M$ and therefore is valid. Since $x$ represent $\succeq$ and every type in $M$ reciprocates, $\delta^{\prime}$ is weakly increasing in both arguments. Finally, Lemma 9 and the validity of $M$ imply $\left(K, \delta^{\prime}\right)$ is increasing.

\subsection{Proof of Theorem 6}

Verifying the validity of $M_{k}^{0}$ and $M_{k}^{1}$ is straightforward. To prove the converse, let $\left(T^{\prime}, \delta^{\prime}\right)$ be a valid binary model such that $\delta^{\prime}(x, y) \in\{0,1\}$ for all $x, y \in T^{\prime}$ and consider the mapping $\eta_{\delta}: T^{\prime} \rightarrow \mathcal{C}\left(T^{\prime},\{0,1\}\right)$ defined by $\eta_{\delta}(x)(y)=\delta^{\prime}(x, y)$. Since $\delta^{\prime}$ is continuous, so is $\eta_{\delta^{\prime}}$. Hence, $\eta_{\delta}\left(T^{\prime}\right)$ is compact. By Lemma 3(ii), this means that $\eta_{\delta}\left(T^{\prime}\right)$ is compact when $\mathcal{C}\left(T^{\prime},\{0,1\}\right)$ is endowed with the sup metric $d_{*}$. Choose $0<\epsilon<1 / 2=d(0,1)$ and let $B_{\epsilon}(p)=\left\{q \in \eta_{\delta}\left(T^{\prime}\right) \mid d_{*}(p, q)<\epsilon\right\}$. Clearly, $B_{\epsilon}(p)$ is an open cover of $\mathcal{C}\left(T^{\prime},\{0,1\}\right)$ and therefore, a finite collection of such sets must also cover $\mathcal{C}\left(T^{\prime},\{0,1\}\right)$. Since each set $B_{\epsilon}(p)$ contains exactly one element $p$, we conclude that $\mathcal{C}\left(T^{\prime},\{0,1\}\right)$ is finite. Then, the validity and the symmetry of $\left(T^{\prime}, \delta^{\prime}\right)$ imply that $T^{\prime}$ is finite (otherwise, we would have distinct $t, t^{\prime} \in T^{\prime}$ such that $\left.\delta^{\prime}(t, \cdot)=\delta^{\prime}(\cdot, t)=\delta^{\prime}\left(\cdot, t^{\prime}\right)=\delta^{\prime}(t, \cdot)\right)$.

Then, without loss of generality, assume $T^{\prime}=\{1, \ldots, m\}$. We will prove the result by induction. If $m=1$, the result is obvious. Suppose that the result is true for $m=k$ and assume $m=k+1$ and hence $T^{\prime}=\{1, \ldots, k+1\}$. Validity ensures that $\delta^{\prime}(t, \cdot)$ is constant for some $t \in T^{\prime}$. Suppose this constant is 1 and $t=k+1$. (The proof for the case in which this constant is 0 is symmetric and will be omitted.) Let $T=T^{\prime} \backslash\{t\}$ and let $\delta$ be the restriction of $\delta^{\prime}$ to $T \times T$. 
First, we argue that $(T, \delta)$ must be a valid binary symmetric IPM. If not, there exists some partition, $\mathcal{D}$, other than the finest that such that $\delta\left(t^{\prime}, D\right)=\delta\left(t^{\prime \prime}, D\right)$ for all $D, D^{\prime} \in \mathcal{D}$ and $t^{\prime}, t^{\prime \prime} \in D^{\prime}$. Then, let $\mathcal{D}^{\prime}=\mathcal{D} \cup\{\{t\}\}$ and note that $\mathcal{D}^{\prime}$ proves that $\left(T^{\prime}, \delta^{\prime}\right)$ is not valid, a contradiction. Hence, by the inductive hypothesis, we can assume that $T=\{1, \ldots, k\}$ and either $\delta=\delta_{k}$ or $\delta=\delta_{k+1}$. In the former case, we have $\delta^{\prime}(k, t)=\delta(k+1, t)=1$ for all $t \in T$ and hence $\delta(k, \cdot)=\delta(k+1, \cdot)$, contradicting the validity of $\left(T^{\prime}, \delta^{\prime}\right)$. In the latter case, we have $\left(T^{\prime}, \delta^{\prime}\right)=M_{k+1}^{0}$ as desired. 
Acknowledgments: This research was supported by grants SES9911177, SES0236882, SES9905178 and SES0214050 from the National Science Foundation. We thank Stephen Morris and Phil Reny for their comments. We thank Nicholas Butler for valuable research assistance. 


\section{References}

Aumann, R. (1976): “Agreeing to Disagree," Annals of Statistics, 4, 1236-39.

Bacharach, M. (1985): "Some Extensions of a Claim of Aumann in an Axiomatic Model of Knowledge," Journal of Economic Theory, 37, 167-90.

Battigalli, P. and M. Dufwenberg (2009) "Dynamic Psychological Games," Journal of Economic Theory, 144(1), 1-35.

Battigalli, P. and M. Siniscalchi (2003): "Rationalization and Incomplete Information," The B.E. Journal of Theoretical Economics, 3(1).

Berg, J., Dickhaut, J. and K. McCabe (1995) "Trust, Reciprocity and Social History," Games and Economic Behaviour, 10, 122-42.

Bergemann, D. and S. Morris (2007) "Strategic Distinguishability with an Application to Virtual Robust Implementation," mimeo, Princeton University.

Bergemann, D. and S. Morris (2009) "Robust Virtual Implementation," Theoretical Economics, 4(1), 45-88.

Bergemann, D. and S. Morris and S. Takahashi (2010) "Interdependent Preferences and Strategic Distinguishability," mimeo, Princeton University.

Blount, S. (1995), "When Social Outcomes Aren't Fair: The Effect of Causal Attributions on Preferences," Organizational Behavior and Human Decision Processes, 63, 131-44.

Bolton, G. and A. Ockenfels (2000) "EEC - A Theory of Equity, Reciprocity and Competition," American Economic Review, 90, 166-93.

Brandenburger A. and E. Dekel (1993): "Hierarchies of Beliefs and Common Knowledge," Journal of Economic Theory, 59, 1993, 189-98.

Brown, A. and C. Pearcy (1995) An Introduction to Analysis, Springer-Verlag, New York.

Camerer, C. and A. Thaler (1995): "Ultimatums, Dictators and Manners," Journal of Economic Perspectives, 9, 209-19.

Cave, J. A. K. (1983): "Learning to Agree," Economics Letters, 12(2), 147-52.

Charness, G. and M. Rabin (2002) "Understanding Social Preferences with Simple Tests," Quarterly Journal of Economics, 117, 817-69.

Cox, J. C., D. Friedman and S. Gjerstad (2007) "A Tractable Model of Reciprocity and Fairness," Games and Economic Behavior, 59(1), 17-45.

Dal Bo, P. (2005) "Cooperation under the Shadow of the Future: Experimental Evidence from Infinitely Repeated Games," American Economic Review, 95(5), 1591-1604. 
Dekel, E., D. Fudenberg and S. Morris (2006a) "Topologies on Types," Theoretical Economics, 1, 275-309.

Dekel, E., D. Fudenberg and S. Morris (2006b) "Interim Rationalizability," Theoretical Economics, 2, 15-40.

Dohmen, T., Falk, A., Huffman, D. and U. Sunde (2009) "Homo Reciprocans: Survey Evidence on Behavioral Outcome," The Economic Journal, 119, 592-612.

Dufwenberg, M. and G. Kirchsteiger (2004) "A Theory of Sequential Reciprocity," Games and Economic Behavior, 47, 268-98.

Ely, J. and M. Peski (2006) "Hierarchies of Beliefs and Interim Rationalizability," Theoretical Economics, 1, 19-65.

Falk A., E. Fehr and U. Fishbacher (2008) "Testing Theories of Fairness - Intentions Matter", Games and Economic Behavior, 62(1), 287-303.

Falk A. and U. Fishbacher (2006) "A Theory of Reciprocity," Games and Economic Behavior, 54(2), 293-315.

Fehr E. and K. Schmidt (1999) "A Theory of Fairness, Competition and Cooperation." Quarterly Journal of Economics, 114, 817-68.

Geanakoplos J., D. Pearce and E. Stacchetti (1989) "Psychological Games and Sequential Rationality," Games and Economic Behavior, 1, 60-80.

Geanakoplos, J., and H. Polemarchakis (1982) "We Can't Disagree Forever," Journal of Economic Theory, 28, 192-200.

Jaffray, J-Y., (1975) "Existence of a Continuous Utility Function: an Elementary Proof," Econometrica, 43, 981-3.

Levine, D, (1998) "Modeling Altruism and Spitefulness in Game Experiments," Review of Economic Dynamics, 7, 348-52.

Mariotti, T., Meier, M and M. Piccione (2005) "Hierarchies of Beliefs for Compact Possibility Models," Journal of Mathematical Economics, 41(3), 303-324.

Mertens J.F. and S. Zamir (1985) "Formulation of Bayesian Analysis for Games with Incomplete Information," International Journal of Game Theory, 14, 1-29.

Parikh, R. and P. Krasucki, (1990) "Communication, Consensus, and Knowledge," Journal of Economic Theory, 52, 178-89

Rabin, M., (1993) "Incorporating Fairness into Game Theory and Economics," American Economic Review, 83, 1281-302.

Segal, U. and J. Sobel (2007) "Tit for Tat: Foundations of Preferences for Reciprocity in Strategic Settings," Journal of Economic Theory, 136(1), 197-216. 
Sobel, J. (2005) "Interdependent Preferences and Reciprocity," Journal of Economic Literature, 43(2), 392-436. 